\title{
On the continuity of thought and the representation of knowledge: Electrophysiological and behavioral time-course measures reveal levels of structure in semantic memory
}

\author{
JOHN KOUNIOS \\ University of Pennsylvania, Philadelphia, Pennsylvania
}

\begin{abstract}
Time-course studies of semantic verification are reviewed, discussed, and reinterpreted with the aim of drawing general theoretical conclusions about semantic memory structure. These reaction time, speed-accuracy tradeoff, speed-accuracy decomposition, and event-related (brain) potential (ERP) studies suggest that semantic memory is structured on at least three levels. In particular, specific models of the intermediate (macrostructural) level are discussed and compared. ERP investigations of this level suggest that context-independent and context-dependent types of semantic information are potentially isolable and analyzable.
\end{abstract}

Research on semantic memory has been one of the mainstays of cognitive psychology for more than two decades. Since the early papers of Collins and Quillian (1969) and Meyer (1970), the focus of this research has been on exploring how people represent, access, and utilize semantic information about natural categories. Of these interests, the representation of semantic information has been the primary concern. Indeed, the investigations of the 1970s pioneered the use of a number of ideas that have proved important to the study of knowledge representation, such as networks, features, exemplars, fuzzy sets, and so on (for reviews, see Chang, 1986; Kintsch, 1980; E. E. Smith, 1978).

\section{Experimental Approach}

The basic experimental approach taken by this body of work is, in principle, simple. Subjects are presented with pairs of terms representing common concepts, usually in the form of a sentence (e.g., "All dogs are animals," or "Some people are trees"). In such a sentence verification task, subjects must quickly decide whether the semantic relation given by each sentence is correct or incorrect and respond to each stimulus with a rapid, accurate buttonpress to indicate their decision. Since the sentences are typically rather simple, accuracy is usually high. So, reaction time (RT) has been the dependent variable of primary interest.

The preparation of this article was supported by National Institute of Mental Health Grant CEP 1 R29 MH45447-01A3 to J.K. Thanks go to Jane Anderson, Phillip J. Holcomb, James Neely, Allen Osman, Richard Schweickert, Roderick Smith, and various anonymous reviewers for helpful discussions, advice, and comments. J.K. is affiliated with the Institute for Research in Cognitive Science at Penn. Correspondence should be addressed to J. Kounios, Department of Psychology, University of Pennsylvania, 3815 Walnut St., Philadelphia, PA 19104-6196.

\section{Rationale}

The logic of this enterprise is straightforward. It was hoped that comparing mean RTs to syntactically similar sentences embodying different semantic relations between the subject and predicate terms would allow inferences to be made about the format and organization of information in semantic memory. This is a different approach from that taken by most researchers in the field of categorization. These researchers have usually adopted a variety of rating and judgment tasks to study how people form and utilize different types of concepts (for a recent review, see Komatsu, 1992). The latter approach has the advantage of reflecting how people learn and use categories. The drawback is that it is difficult to infer in this way the structure or format of this knowledge as it is stored in semantic memory, since rating and judgment tasks invoke undetermined processing mechanisms that can transform and obscure important characteristics of stored knowledge. This is why semantic memory researchers have preferred the sentence verification paradigm. RTs to these regimented sentences are fairly quick, and subjects are, at best, only dimly aware of how they go about verifying such propositions. The implication is that the processing mechanisms that allow a subject to verify these sentences are simple, experimentally controllable, and probably understandable. Speeded sentence verification might therefore allow researchers to penetrate through these processing mechanisms to the underlying knowledge representations.

\section{Critique of Semantic Memory Research}

Unfortunately, by the late 1970 s it became clear that this enterprise might not be so straightforward. A number of impressive models of semantic memory had been proposed and put into competition with each other. But this "Darwinian" approach did not prove successful. Though 
each of these models had its own set of strengths and weaknesses (Chang, 1986), there was no clearly superior model to survive and dominate the field. In fact, in discussing semantic memory, many current introductory cognition texts frequently describe some of the earliest of these models (e.g., Collins \& Quillian, 1969; E. E. Smith, Shoben, \& Rips, 1974) as exemplifying the field. What went wrong? There are at least two possibilities.

Blurring of structure and process. The most obvious difficulty with past semantic memory research is the familiar structure versus process issue (Anderson, 1978; Chang, 1986; Hollan, 1975; Kounios, 1993; Kounios \& Holcomb, 1992; Kounios, Montgomery, \& R. W. Smith, 1994; Kounios, Osman, \& Meyer, 1987; Rips, E. E. Smith, \& Shoben, 1975; E. E. Smith, 1978; E. E. Smith et al., 1974). In brief, this problem concerns whether the accumulated database of semantic verification results is best explained in terms of complex knowledge structures and relatively simple processing mechanisms (see, e.g., Collins \& Quillian, 1969; Glass \& Holyoak, 1975) or relatively simple knowledge representations and more complex processing mechanisms (see, e.g., McCloskey \& Glucksberg, 1979; Ratcliff \& McKoon, 1982; E. E. Smith et al., 1974). To oversimplify a bit, this is another way of asking whether people verify propositions by simply looking up something that is already directly stored in semantic memory, or whether they must infer or compute the truth of a statement on the basis of information stored in a more primitive form. Answering this question on the basis of RT verification studies has proved a daunting task (Chang, 1986; Kounios \& Holcomb, 1992; Kounios et al., 1987).

Theorists have taken a variety of approaches in dealing with the structure/process question. Since the mid-1970s, the most common approach has been to assume or assert that there is little or no structure to stored semantic information. For instance, supporters of sequential-sampling feature-comparison models assume minimal structure, or even ignore the question of structure (e.g., McCloskey \& Glucksberg, 1979; Ratcliff \& McKoon, 1982). The parallel distributed processing (PDP) approach (Rumelhart, McClelland, \& the PDP Research Group, 1986) is based on the notion that stable knowledge structure is not stored; it is a statistical abstraction embodied in the weights on links connecting primitive featural units. Similarly, Barsalou (1987) argued that there are no stable stored categories: "Invariant representations of categories do not exist in human cognitive systems. Instead, invariant representations of categories are analytic fictions created by those who study them" (p. 114). Barsalou argued that categories are constructed on demand (influenced by contextual considerations) from primitive, relatively unstructured, stored information.

Premature detail. A second, more subtle, problem is that the initial strategy used to investigate semantic memory was arguably too ambitious. Initially, a number of specific models were proposed and tested with varying outcomes. However, the things that made these models so interesting, namely their detail and specificity, were probably premature. Substantial theoretical detail was included in many of the best known models in an attempt to extend the range of empirical phenomena with which they could cope. Unfortunately, this theoretical "sufficiency" was achieved at the expense of theoretical "transparency" (see E. E. Smith, 1978).

However laudable the goal, such premature detail had the effect of rendering empirical tests less informative than they might have been. This point is not unique to semantic memory research. In the conclusion of a review paper on mental chronometry and models of human information processing, Schweickert and Boggs (1984) lamented the slow pace of progress in that field (perhaps not coincidentally at about the same time that Chang, 1986, attempted to summarize, evaluate, and salvage as much as possible from the semantic memory corpus). They cited and endorsed Broadbent's (1958) proscription against premature theoretical detail, because "if a detailed theory is falsified, in itself this is not progress unless the set of remaining theories is notably smaller as a result. He suggests that the optimal strategy is to ask questions so that each answer reduces the number of remaining theories by half"'(Schweickert \& Boggs, 1984, p. 272). They pointed out that this approach contrasts with that described by Newell (1973) in a paper revealingly entitled "You Can't Play 20 Questions With Nature and Win." Schweickert and Boggs sided with Broadbent in this methodological/philosophical dispute. As for semantic memory, most of the published experimental results seem more geared to test specific aspects of specific models rather than to excising significant portions of the model-space. So it can be argued that the history of research in this area represents a case study in the risks of premature theoretical detail. This dilemma clearly implies that researchers should, at least for the time being, focus their experimentation on general questions applicable to whole classes of theories rather than to issues peculiar to specific models.

\section{Overview}

In this spirit, general theoretical conclusions about semantic memory structure will be drawn from the following discussion and reinterpretation of selected studies. ${ }^{1}$ Although the methodological approaches taken by these studies vary considerably, a common thread binds them together - namely, that they all involve investigating the time course of information processing in semantic verification tasks. In what follows, it is argued that available time-course methods do shed light on the structure versus process issue, and that such studies indicate that semantic memory is, in fact, highly structured on several levels. In addition, specific research strategies for distinguishing stable semantic memory structure from contextually influenced conceptual representations are discussed. These strategies may enable researchers to investigate the nature of this structure. 


\section{CONVENTIONAL TIME-COURSE STUDIES OF SEMANTIC VERIFICATION}

\section{Reaction Time Studies}

One approach to studying the time course of semantic verification is to measure $\mathrm{RT}$ in a priming paradigm (Lorch, 1982; Rosch, 1975). In this approach, the stimulus onset asynchrony (SOA) between a category and a subsequent exemplar term is systematically varied across a range of values and the effects on RT and accuracy are examined. The most clear-cut example of this approach was provided in a study by Lorch (1982, Experiment 1 ) in which he simultaneously manipulated a measure of strength of association between the category and exemplar terms (i.e., instance dominance; see Battig \& Montague, 1969) and the category-exemplar SOA for the purpose of testing alternative predictions of various classes of spreading-activation models of semantic memory. He found that strength of association influenced priming in such a way that RT to verify low instancedominance category-exemplar pairs was longer than the RT to verify high instance-dominance pairs. However, there was no significant interaction between the effects of SOA and instance dominance on RT, suggesting that the priming functions (for low and high instance-dominance pairs) describing RT as a function of SOA were parallel. In other words, strength of association and SOA apparently had additive effects on mean RT. Lorch interpreted these results to mean that strength of association determines the asymptotic level of activation achieved on a trial, but not the duration of an early activation process. ${ }^{2}$ According to additive-factors logic (Sternberg, 1969) this pattern indicates that these factors influence independent stages of processing. ${ }^{3}$

Hampton (1984) compared RTs to verify category membership (e.g., oak-is a tree) and property (e.g., sparrowhas wings) statements across three levels of predicate production frequency (a measure of association strength similar to instance dominance). He found apparently additive effects of type of relation (category vs. property) and production frequency (low, medium, and high) on verification RT. Again, according to additive-factors logic, these results are consistent with the notion that type of relation and production frequency affect independent processing stages. These results are also consistent with the notion that strength of association affects a comparatively early encoding/retrieval stage that is independent of a later decision stage whose duration is influenced by the type of relation being verified.

More recently, Thomsen, Lavine, and Kounios (in press) used a sentence verification paradigm in order to study the organization of social values (e.g., freedom) and attitudes (e.g., pro-choice) in semantic memory. Each stimulus sentence was of the following form: "The concept of [value/attitude] is closely related to the concept of [attitude/value]." Thus, the task involved a relatedness rather than a class-inclusion judgment. The important independent variables were order (i.e., value-attitude or attitude-value priming conditions) and subject/predicate

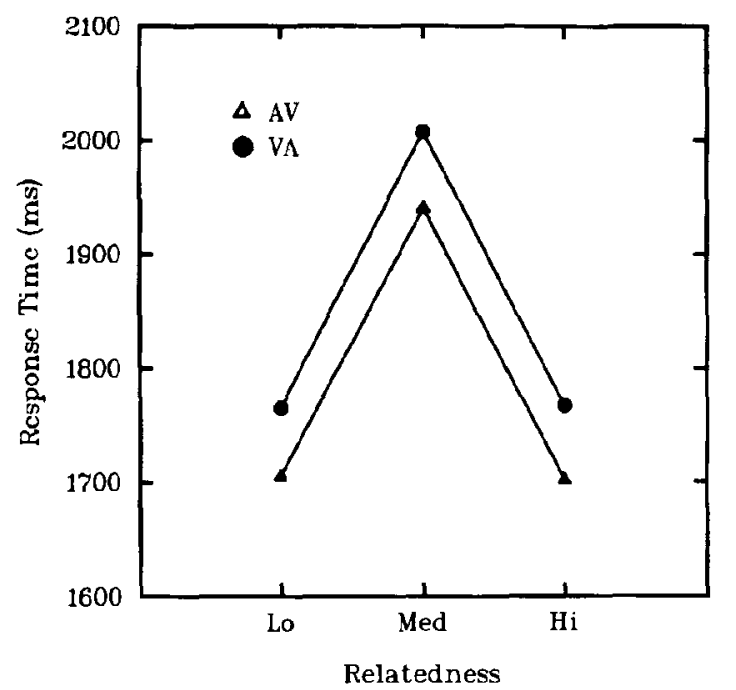

Figure 1. Additive inverted-U reaction time (in milliseconds) functions resulting from the manipulation of attitude-value relatedness and order (VA, value-attitude; $A V$, attitude-value) in the verification study of Thomsen, Lavine, and Kounios (in press).

relatedness (as assessed by a 7-point scale administered to the same subjects after completing the RT study). As Figure 1 shows, the graph of RT as a function of (questionnaire) relatedness depicts an inverted- $U$ shaped function (cf. E. E. Smith et al., 1974): Sentences containing a subject and predicate of moderate relatedness yielded the slowest RTs, while sentences containing terms of high and low relatedness yielded much faster RTs. In addition, sentences in which an attitude was the subject and a value was the predicate yielded RTs that were faster than RTs for sentences of the opposite concept order. Most importantly, order and relatedness had apparently additive effects on RT. According to additive-factors logic, order and relatedness therefore influence independent processing stages. This interpretation was further supported by the finding that the order effect vanished when the number of close associates (i.e., the number of value or attitude terms judged by subjects to be closely related to a given value or attitude term) was statistically controlled for (suggesting a fan effect, Anderson, 1983). Along with the results of Lorch (1982) and Hampton (1984), the results of Thomsen et al. (in press) are consistent with the notion that there is a comparatively early stage responsible for encoding/retrieval followed by a decision mechanism responsible for judging the truth of the sentence. In this case, the hypothesized early encoding/retrieval stage is presumably influenced by priming order, while the later decision stage may be influenced by subjectpredicate relatedness (cf. E. E. Smith et al., 1974).

\section{Speed-Accuracy Tradeoff}

Another important chronometric technique that has been applied to the study of semantic verification is the speed-accuracy tradeoff (SAT) method (Casey \& Heath, 1990; Corbett \& Wickelgren, 1978; Ratcliff \& McKoon, 
1982). Although several versions of this technique exist (see the reviews by Pachella, 1974; Wickelgren, 1977), they all embody the same underlying principle: Instead of examining differences in processing time (i.e., RT) as a function of experimental manipulations, they measure response accuracy as a function of the amount of processing time the subject takes (or is allowed to take). By measuring response accuracy at a number of levels of processing time, an SAT function describing the time course of the accrual of accuracy can be derived. This technique yields three parameters of particular theoretical interest: the point in time at which response accuracy or information starts to rise above chance, the rate at which this information approaches its final asymptotic level, and the asymptotic level of accuracy itself.

SAT and category verification. Corbett and Wickelgren (1978) examined these parameters for categoryexemplar pairs varying in instance dominance. They found that the time at which information about the correct response started to rise above chance was unaffected by the manipulation of instance dominance, as was the rate at which this information approached its asymptotic level. However, the asymptote itself was significantly higher for exemplars higher in instance dominance. Corbett and Wickelgren interpreted these results to mean that retrieval dynamics (i.e., the rate of retrieval of semantic information from memory) is not affected by the manipulation of instance dominance, but that the asymptotic strength of the association between a category-exemplar pair does vary with this manipulation.

In their discussion of Corbett and Wickelgren's (1978) results, Casey and Heath (1990) focused on the bothersome quality of this finding. Why should asymptotic accuracy be lower for category-exemplar pairs that are lower in instance dominance? For example, why should subjects be less accurate when judging that, say, a spider is an animal, than when judging that a dog is an animal? After all, a spider is just as much an animal as is a dog.

Casey and Heath (1990) had the idea that the differences in asymptotic accuracy obtained by Corbett and Wickelgren (1978) could be due to the inclusion of exemplars of dubious category membership (i.e., very low typicality-e.g., is a radio a piece of furniture?) in the low instance-dominance pairs (see note 2) ${ }^{4}$ Subjects might legitimately brand some of these pairs as being invalid, resulting in an "incorrect" response. This could be the cause of the lower asymptotic accuracy for low instancedominance pairs. Casey and Heath tested this hypothesis by replicating Corbett and Wickelgren's experiment, including some uncertain category-exemplar pairs in the low instance-dominance condition of their study, and using only low instance-dominance pairs of certain category membership in a parallel condition (i.e., the way Corbett \& Wickelgren should, in hindsight, have done it).

Casey and Heath's (1990) results confirmed their interpretation of Corbett and Wickelgren's (1978) study. When very low typicality exemplars were included, asymptotic accuracy (but not the rate of approach to the asymptote) was reduced. However, when none of the lower typicality exemplars were of uncertain category membership, asymptotic accuracy was unaffected by instance dominance, though the rate of approach to this asymptote was lower for the lower typicality stimulus pairs.

However, Casey and Heath (1990) obtained another interesting and theoretically significant empirical finding (the implications of which they did not discuss) that replicated one of Corbett and Wickelgren's (1978) results. In both SAT studies, it was found that the zero-sensitivity intercept of the fitted SAT functions was not discernibly affected by differences in typicality. In other words, typicality does not seem to affect the point in time at which information about the correct response starts to grow. This finding is reminiscent of the additive-factors studies discussed above in that it suggests that early "upstream" processes during verification vary little, if at all, in duration as a function of instance dominance or typicality. As shall become evident later, this finding is of considerable theoretical importance.

The interpretation of SAT functions. Casey and Heath's (1990) most salient theoretical assertion is that their results support models of semantic verification characterized by a continuous accumulation of response information over the course of a trial (see, e.g., Casey \& Heath, 1989; McCloskey \& Glucksberg, 1979; Ratcliff \& McKoon, 1982). The idea is that the continuous accrual of response accuracy that characterizes their SAT data signifies that the underlying accumulation of response information available to subjects is also gradual or continuous in nature. Within the context of a semantic verification experiment, this interpretation is very natural and intuitive, as several of the major semantic memory models posit mechanisms by which sets of features or attributes corresponding to the category and exemplar terms are compared. Each comparison of a pair of features yields a small increment in information about the correct response. The comparisons occur either sequentially or in parallel, with different starting or finishing times (or both). The result is either a continuous response accuracy during a trial or a "quasi-continuous" accumulation characterized by a large number of tiny steps.

Unfortunately, this sort of interpretation of SAT data is unjustified (Kounios et al., 1987; Meyer, Irwin, Osman, \& Kounios, 1988; Wickelgren, 1977). For instance, suppose that on each individual trial, response information did not accrue gradually, but in a single discrete jump from a state of no information about the correct response to the final state of complete accuracy. If, because of variability in the stimuli and the fluctuating states of the subject, the moment in time of this transition varies from trial to trial, then the SAT function obtained by averaging the data over trials (and subjects) will be continuous, even if the underlying mechanism yields a discrete allor-none transition on each individual trial. Wickelgren (1977) pointed out this limitation on the kinds of theoretical interpretations that can be given to SAT curves and lamented the unlikelihood of discovering an experimental technique that could reveal whether the underlying mechanisms really yield continuously accumulating 
response information, or whether such decisions are made discretely.

\section{Speed-Accuracy Decomposition}

Fortunately, Meyer and his associates (Meyer et al., 1988) developed a technique that sheds light on this issue, which they called "speed-accuracy decomposition" (SAD; see also R. W. Smith, Kounios, \& Osterhout, 1996). SAD combines a new experimental procedure and quantitative analysis that enables the experimenter to remove from an SAT function the contribution of trials on which subjects responded from the final state of relatively complete response accuracy. The resulting speed-accuracy function quantifies any partial information that may exist, that is, information about the correct response that is available from incomplete processing only.

This is accomplished with an experimental procedure that randomly interleaves two types of trials: (1) conventional ("regular") RT trials on which a subject responds as soon as processing has been normally completed, and (2) speed-stress ("response-signal") trials on which a response signal (e.g., a tone) presented at some time lag after the test stimulus indicates that an immediate "best guess" response is required. Because both of these types of trials begin in the same way (i.e., with the presentation of a test stimulus) and are randomly interleaved, the subject must assume that each trial will be a regular one and adopt the mental set of emphasizing accuracy as the primary goal and response speed as a secondary goal. However, if a response signal is presented during the trial, the subject must suddenly reverse priorities, immediately issuing a "best guess" response.

Several different response-signal lags can be randomly intermixed in the same experiment. Plotting the signaltrial response accuracies across these lags yields a conventional SAT function. However, this SAT function (like all others) is contaminated by the inclusion of some trials on which the subject responded seemingly because of the demands of the response signal, but really because he/she had normally completed processing of the test stimulus and was ready to respond on the basis of complete information about the stimulus. In other words, in any SAT experiment, some percentage of the responses are made at particular points in time not because the task demands a guess at that time, but because even if it were a conventional RT task, the subject would have responded at that point in time anyway (i.e., on the basis of completed processing).

This is where the regular-trial RT data come in. They provide an estimate of the distribution of normal finishing times for the verification judgment. These data constitute one component of the statistical mixture comprising the response-signal data (the other component being trials on which responses were made on the basis of incompleted processing, that is, "guesses"). The first step toward disentangling this mixture is to obtain the durations of the guesses (on the basis of an equation whose derivation is given in Meyer et al., 1988). Dura- tion is computed by comparing the signal-trial RT distribution (separately for each signal lag) to the regulartrial RT distribution for each time bin of, say, $20 \mathrm{msec}$. Use of a quantitative "race" model allows the removal of the proportion of trials based on completed processing (estimated from the regular-trial RT distribution) from the signal-trial distribution for each time bin. This yields an estimated RT distribution for guessing responses; that is, responses incited by the response signal and based only on incomplete processing.

The second step is to derive the accuracy of these guesses (on the basis of a separate equation derived in Meyer et al., 1988). Given the first step, this is easily accomplished. The signal-trial accuracy for each lag is assumed (on the basis of plausible and largely verifiable assumptions) to also be a mixture of the accuracies of responses based on complete processing and responses based on incomplete processing. Using the ratio of these two types of processes obtained in the first step, the contribution of normally completed processes (estimated from the regular-trial accuracy for each time bin) is removed from the signal-trial accuracy (for the corresponding time bin). The overall guessing accuracy for that lag can then be computed by calculating a weighted average of the guessing accuracies across all time bins. Plotting guessing accuracy as a function of response-signal time lag yields a partial-information accumulation function describing the accumulation of partial information over the course of the average trial.

SAD and sentence verification. Kounios et al. (1987) explored the application of SAD to semantic verification. They argued that this technique would be a useful tool for resolving aspects of the structure versus process debate. According to their analysis, models of semantic verification fall roughly into two basic classes: network search and feature computation. Network-search models (see, e.g., Collins \& Quillian, 1969; Glass \& Holyoak, 1975) posit that concepts are stored as nodes in an associative network whose links represent the semantic relations between these concepts. When a category-exemplar pair (or a sentence relating a category and an exemplar) is presented for verification, these terms activate their corresponding nodes. A search is undertaken for an associative path connecting these nodes. If such a path is found, the stimulus is judged to be true. If such a path is not found, or if contradictory paths are found, the stimulus is judged to be false. Although the particulars of the various classical network models vary (see Chang, 1986, for a review), they are generally based on the idea that relations between concepts are prestored (E. E. Smith, 1978). Verification therefore usually amounts to a search of the semantic memory structure. In a simple, "pure" search model, the verification process should yield a discrete, all-or-none transition from a state of no information to a state of relatively complete information. This follows from the nature of the search process. The subject should not have any information about the truth of a semantic relation until the search of memory is complete. Once the search 
is finished, complete information becomes available (for details of this rationale, see Kounios, 1993; Kounios et al., 1987; Kounios et al., 1994).

Other models emphasize computation rather than search (see, e.g., Casey \& Heath, 1989; McCloskey \& Glucksberg, 1979; Ratcliff \& McKoon, 1982). These models deemphasize or eliminate the notion of global semantic memory structure, positing that concepts are stored in terms of lists or sets of semantic features. Semantic relations are not prestored, but are computed on demand by comparing pairs of feature sets. As explained above, such models predict gradually accruing response information.

Kounios et al. (1987) pointed out that the empirical status of the structure versus process distinction could be elucidated by SAD. If the technique yields no residual partial information, then some simple search model of semantic memory would be supported. On the other hand, evidence of partial information would favor some variant of a computational model. They therefore applied SAD to the verification of simple sentences relating category and exemplar terms (e.g., "All dogs are animals," "All animals are dogs," or "All dogs are clothes") and, in fact, found evidence of gradually accumulating partial information. This information started to accumulate at about the same point in time regardless of the type of sentence being verified, and grew at an approximately linear rate (in units of $d^{\prime}$ ) that depended on the type of sentence. These results therefore support a featurecomputation interpretation of semantic verification, although other findings (described below) suggest that this is probably not the whole story (Kounios, 1993). In any event, Casey and Heath's (1990) conclusion that response information accumulates gradually in a semantic verification task was apparently correct, though basing this inference on the shapes of SAT functions was incorrect. ${ }^{5}$

Relationship to other time-course studies. There is, however, another important point of comparison between the studies of Casey and Heath (1990) and Kounios et al. (1987). The latter found that partial information started to grow at about the same point in time regardless of the type of relation being verified. They hypothesized that verification consists of at least two (possibly discrete) stages, or "superstages," to use Miller's (1988) term. The early stage is responsible for stimulus encoding and the retrieval of relevant semantic information. The second stage accomplishes computational verification in a manner that yields usable partial response information. Kounios et al. observed that since the duration of the early stage is not discernibly affected by changes in the type of class-inclusion relation, these upstream processes seem to take about the same amount of time irrespective of the type of stimulus. This suggests that the corpus of RT findings obtained over the last two decades (see Chang, 1986) reflects effects on downstream decision processes, that is, processes that actually compute the truth value of the retrieved (or constructed) semantic relation. It is more than suggestive that Corbett and Wickelgren (1978) and Casey and Heath (1990) have both found that other semantic variables (viz., instance dominance and typicality) also had no apparent effect on the time of onset of information accrual observed with a different experimental technique (SAT). When these findings are considered together with Lorch's (1982) priming results suggesting that an early activation process is unaffected by the manipulation of instance dominance (along with the similar results by Hampton [1984] and Thomsen et al. [in press] described earlier), the notion that early processes in a verification task do not significantly vary in duration is supported by data obtained with three different experimental procedures and considerable variation in stimuli. 6 As will become apparent later, a fourth - and radically different - type of data suggests the same conclusion.

The granularity of semantic relations. A recent study by Kounios et al. (1994) similarly applied SAD to the investigation of semantic relations. According to the theoretical analysis of Chaffin and Herrmann (1988), there are two general ways to characterize semantic relations in memory: (1) They can be thought of as unitary links between concept nodes in a network, or (2) they can be thought of as complex concepts in their own right, in which case they should be decomposable into semantic features (or "relation elements," to use Chaffin \& Herrmann's term), as are noun concepts.

Following a suggestion by Glass and Holyoak (1975), Kounios et al. (1994) focused their investigation on the relation of ownership or possession, because this relation is very different from the kinds of class-inclusion relations typically used in verification experiments, and because ownership statements cannot be verified by a similarity heuristic (cf. E. E. Smith et al., 1974). For instance, the truth of the statement "Many people own computers" is unrelated to the similarity of people to computers. Glass and Holyoak argued that an ownership relation is probably verified by retrieving a unitary ownership link between semantic network nodes corresponding to the subject and predicate terms of such a sentence. Kounios et al. (1994) argued that if this were the case, the verification of such sentences should not yield partial information, because such a unitary link would have to be retrieved in an all-or-none fashion. However, if semantic relations are themselves complex concepts decomposable into elements (as Chaffin \& Herrmann argued), then an SAD study utilizing ownership sentences should, in fact, yield partial information. Kounios et al. did, in fact, find partial information, thereby supporting the view that relations, as well as noun concepts, are granular rather than being discrete, unitary entities. Furthermore, their results are consistent with the notion that partial information (in units of $d^{\prime}$ ) accumulates in an approximately linear fashion over time right up to the level of information exhibited on regular trials. Kounios et al. argued that such a linear accumulation would be consistent with a model based on the retrieval or comparison of relational features at a relatively constant rate over time.

Boundary conditions. The SAD studies cited above indicate that stored semantic representations, whether of noun or relational concepts, are composed of small-grain (e.g., featural) information. This, however, might not al- 
ways be the case. A study by Kounios (1993) focused on an ambiguous finding from the original Kounios et al. (1987) article. The data from the 1987 report exhibited a peculiar pattern of partial-information accumulation. This information grew to high levels of sensitivity at late response-signal lags. However, this growth was much too slow relative to the speed and accuracy of regulartrial RT responses to predict the latter data. In other words, extrapolation of the partial-information accumulation functions to the level of accuracy exhibited on regular trials predicted regular-trial RTs that were slower and/or less accurate than those that were actually obtained.

Kounios et al. (1987) considered two classes of models to explain this phenomenon. The first was a singleprocess computational model (after simulations by Ratcliff, 1988) that predicts a reduction in the slope of a partial-information accumulation function during the latest phase of a trial because of noise in the system and the relative weakness of slower processes (i.e., some trials are slower because they utilize slower, weaker processes that yield lower accuracy). The other was a dualprocess model combining independent fast-search and slow-computational mechanisms racing in parallel, the first one to successfully provide a verification judgment determining the response. In the latter model, the computational mechanism yields gradually accruing partial information from feature comparisons, while the search mechanism yields no partial information because it simply looks up discrete, stored relations represented in a semantic network. Since the hypothetical search yields no partial information, and since the regular-trial RT results consist of a mixture of responses - those based on fast search and those based on slow computation-the regular-trial RTs should seem faster and/or more accurate than would be predicted on the basis of the partialinformation accumulation functions alone (because the available partial information is generated by the slow computational process only).

The most important difference between these two approaches is that the dual-process model posits the existence of a searchable semantic memory structure, while the single-process model assumes no such structure. Consequently, evidence favoring the dual-process model would also support the notion that semantic memory might also contain structured coarse-grain information (e.g., unitary relations, or perhaps even propositions) in addition to the fine-grain (featural) information.

Kounios (1993) argued that if the dual-process model were correct, then, in principle, experimental factors might differentially affect the two processes. In this case, such experimental factors could produce different effects on the quickest (i.e., search-based) and slowest (i.e., computation-based) RTs in a conventional sentence verification (RT) task. In fact, he found such evidence, suggesting that while the fundamental informational units of semantic memory may be of small grain size, larger-grain information is apparently also present and may be utilized in certain situations. In contrast, the SAD study of ownership statements by Kounios et al. (1994) indicated that linear extrapolation of the partial-information accumulation function nicely predicts the RT and sensitivity exhibited on regular trials, suggesting that subjects were tapping only fine-grain information while verifying ownership statements. This suggests that relational information is, in general, stored only in fine-grain form, or that this may be a peculiarity of the ownership relation in particular.

The discovery of all-or-none information processing. More recently, R. W. Smith and Kounios (in press; see also Kounios \& R. W. Smith, 1995) attempted to directly demonstrate the existence of discrete all-or-none information processing. In their first experiment, they employed an anagram lexical decision task in which a (nonword) string of letters is presented and the subject's task is to determine whether any combination of the letters in the string yields a legal word or not. At the earlier of the two signal lags Smith and Kounios employed, they found a tiny but statistically significant trace of partial information (possibly due to heuristics that subjects may have employed on a small number of trials), while at the later lag there was no discernible partial information.

In Smith and Kounios's (in press). second experiment, they sought to vary this paradigm so as to yield substantial levels of partial information. Since previous studies had demonstrated that semantic judgments yield partial information, Smith and Kounios combined the anagram task with a semantic judgment task. In this new paradigm, each letter string could be rearranged to form a word. The subject's task was to determine whether the scrambled word presented on the screen referred to a highly imageable object or not. Surprisingly, this task yielded no discernible evidence of partial information at either of the two signal lags employed. Contrary to expectations, this second experiment provided even firmer evidence of discrete all-or-none processing than did the first. (See Smith et al., 1996, for simulations that demonstrate the reliability and robustness of these findings.)

The anagram results of Smith and Kounios (in press) have two important implications for investigations of discrete and continuous representation and processing in semantic memory. First, they demonstrate that all-or-none processing does indeed exist and can be detected by SAD, which supports the applicability of this technique to the study of semantic memory. Second, they indicate, in contrast to previous results, that semantic judgments (here based on stored imagistic information) may be accomplished in an all-or-none fashion, which suggests discrete knowledge representation. Unfortunately, the latter implication must, at present, remain tentative because it will require further research in order to answer certain important questions about the processing strategy subjects employed in the anagram-imageability task. For instance, they may have used imageability information to narrow the range of legal-word candidates prior to solving the anagram. In this case, the actual (all-or-none) solution of the anagram would have occurred last, yielding no par- 
tial information. Clearly, further SAD investigations of this phenomenon are in order.

\section{Conclusions}

Time-course studies such as those reviewed above utilize valuable methods capable of shedding considerable light on issues of long-standing interest to semantic memory researchers. In particular, these studies suggest two important conclusions: (1) Differences in verification RT across the various types of sentences or categorical relations are usually due to differences in the duration(s) of one or more late decision processes, with little or no effect on early (e.g., encoding, access, or retrieval) processes. (2) In such tasks, the retrieved information that these decision mechanisms process is mostly small-grain (i.e., probably featural) in nature, although it seems likely that there are situations in which semantic verification is based on larger, more complex units of information.

These conclusions are both enlightening and frustrating. Although the chronometric methods that gave rise to them have made possible a considerable reduction in the semantic memory model space, such studies nevertheless highlight their own inherent limitations. The ultimate goal of semantic memory research has arguably been an elucidation of the format of stored semantic representations rather than an analysis of the (possibly ad hoc) decision processes subjects use to verify sentences. The early access and retrieval mechanisms that provide information for the decision processes should theoretically yield a more direct view of the nature of these representations. However, these early processes are comparatively invisible to conventional behavioral research techniques, because under only limited circumstances do they provide information upon which a verification response can be directly based, necessitating further computation before a decision can be made. Moreover, these early processes seem to vary little, if at all, in their temporal duration, thereby absorbing factor manipulations without discernible reactions. This indicates that the sorts of conventional chronometric methods described above will probably be of limited use in studying these important upstream processes.

RT and speed-accuracy techniques are means of analyzing the latency and accuracy of overt responses to stimuli. In a verification task, these overt responses necessarily indicate a subject's verification decision. Consequently, the most important influences on these responses (as explained earlier) are factors affecting the decision processes. These factors include characteristics of the stored semantic representations (as they influence decision processes), but they also include a host of contextual variables that obfuscate characteristics of the predecision semantic representations (Chang, 1986; Gruenenfelder, 1986; Kounios, 1993; McCloskey \& Glucksberg, 1979). Since these overt responses are, at best, contaminated indicators of semantic structure, what is obviously needed is a dependent measure not determined by the decision processes that drive overt responses. This is where electrophysiology comes in.

\section{THE ELECTROPHYSIOLOGY OF SEMANTIC MEMORY}

\section{Event-Related Brain Potentials}

The human brain continuously generates complex patterns of electrical activity. A subset of this activity can be measured by attaching electrodes to a person's scalp and amplifying the faint electrical signals that penetrate the layers of bone and tissue that separate these electrodes from the brain. This ongoing electrical activity constitutes the electroencephalogram (EEG).

Unfortunately, EEG measurements have not yet proved to be of great use in cognitive psychology. These complex waveforms represent agglomerations of many disparate aspects of brain activity, including lower autonomic functions as well as higher forms of cognition. Consequently, it is difficult to tease apart these numerous sources of electrical activity and their contributions to the overall waveform.

The event-related potential (ERP) technique circumvents many of the problems associated with analyzing the electrophysiology of cognition. In an ERP experiment, EEG waveforms (i.e., graphs of electrical amplitude as a function of time) are measured and recorded from the onset of each stimulus until the end of each experimental trial. These waveforms are then averaged across trials for each condition of the experiment. This averaging serves to wash noise out of the waveform, noise being defined as any electrical activity not associated with the requirements of the experimental task. The resulting ERP waveform depicts the subset of the brain's electrical activity measurable at the scalp that is associated with the processing of the test stimulus and the execution of any decision and response that may be required by the task (Hillyard \& Picton, 1987, have provided an excellent introduction to ERP research).

The ERP waveform is characterized by a series of peaks and valleys called "components." According to standard nomenclature, these components are named after their polarity ( $P$ for positive and $N$ for negative) and either their temporal order or their latency after the onset of the test stimulus. For example, $\mathrm{P} 3$ is the third positive component; $\mathrm{N} 400$ is a negative component peaking about $400 \mathrm{msec}$ after the onset of the test stimulus. The various components are thought to be associated with neural mechanisms underlying cognitive processes ranging from sensation to response execution (Hillyard \& Picton, 1987; Regan, 1989). Though ERP research may still be considered to be in its early stages, much work has already been done to characterize the cognitive processes corresponding to specific ERP components.

\section{$\mathbf{N 4 0 0}$}

Of particular interest to language researchers is the N400 component (see Osterhout \& Holcomb, 1995, for a recent review). In the initial report that sparked interest in the N400, Kutas and Hillyard (1980) measured ERPs to the final words of visually presented sentences that were displayed one word at a time. They found that sen- 
tences ending in a semantically inappropriate final word (e.g., "He spread the warm bread with socks") yielded a negative component peaking about $400 \mathrm{msec}$ after the onset of the final word. In contrast, a sentence ending with a semantically appropriate final word (e.g., "It was his first day at work") yielded little or no N400. Kutas and Hillyard established that the incongruency that incited the $\mathrm{N} 400$ was specifically linguistic or semantic in nature (rather than a general surprise reaction) by also presenting to subjects comparable sentences ending with a physically aberrant word (i.e., in larger type). This manipulation yielded a slower positive component (P560) that was experimentally dissociable from the N400.

In later research, Kutas and Hillyard (1984; Kutas, Lindamood, \& Hillyard, 1984) varied the expectedness of sentence final words. They found that relatively unexpected, but semantically acceptable, final words (e.g., "The bill was due at the end of the hour") yielded larger N400s than did expected endings. Furthermore, anomalous endings that were semantically related to the expected ending (e.g., "The pizza was too hot to drink") yielded N400s that were smaller than those produced by anomalous unrelated endings, but larger than the N400s produced by the expected endings. These results suggested that $\mathrm{N} 400$ reflects both violations of semantic context and associative priming between individual words.

More recently, it has been argued that N400 represents a postlexical integrative process that combines outputs of earlier, lower level processes into a higher level representation (Holcomb, 1993; Osterhout \& Holcomb, 1995; Rugg, 1990; Rugg, Furda, \& Lorist, 1988). For example, Brown and Hagoort (1993) performed a lexical decision experiment in which the target words were preceded by semantically related or unrelated primes exposed for durations too brief to permit their perceptual identification (i.e., by quick masking). They argued that any evidence of a semantic priming effect of such briefly displayed primes on N400s to the target words would indicate that N400 reflects automatic spreading activation processes. On the other hand, the failure to find such a priming effect on N400 would indicate that N400 reflects only controlled, postlexical integrative mechanisms (Neely, 1991). In fact, Brown and Hagoort (1993) found that masked primes yielded no N400 priming effect, in contrast to the significant priming effect on lexical decision RT and the significant N400 priming effect obtained for unmasked primes (see also Holcomb, 1988, 1989, 1993; McCarthy \& Nobre, 1993).

\section{ERPs and Sentence Verification}

Fischler and his colleagues (e.g., Fischler, Bloom, Childers, Roucos, \& Perry, 1983; see Fischler \& Raney, 1991, for a review) were the first to measure ERPs during a sentence verification task. They found that predicate N400 was large when the subject and predicate terms of the sentence were dissimilar (e.g., "A canary is a rock," or "A canary is not a rock") relative to the case in which the terms were similar (e.g., "A canary is a bird"). Moreover, the magnitude of N400 did not seem to be particularly sensitive to the truth of the sentence (as indicated by the first two example sentences), but only to the relation between the subject and predicate terms.

Given what is known about ERPs, it would seem that this technique should be a useful tool for investigating semantic memory structure. The fact that the generation and measurement of ERPs do not necessarily depend on the subject making a decision and executing an overt response raised the possibility that aspects of the ERP waveform may reflect the predecision semantic memory processes of particular interest. In particular, Fischler et al.'s (1983) finding that N400 amplitude reflects the relationship between the subject and predicate terms of a sentence without reflecting the sentence's truth value (even when the task requires a truth judgment to be made) suggests that $\mathrm{N} 400$ (and possibly other components) may be an indicator of predecision mechanisms (e.g., retrieval) that could yield clues about semantic memory structure.

Consequently, Kounios and Holcomb (1992) extended the earlier work of Fischler and his colleagues by measuring ERPs during the verification of a broader range of sentences. In addition to manipulating the relatedness of the subject and predicate terms (e.g., dog/animal vs. dog/ furniture), they also manipulated the hierarchical level of these terms (e.g., dog/animal or dog/furniture vs. animal/dog or furniture/dog) and the quantifier applied to each sentence (e.g., "All/Some/No dogs are animals").

In Experiment 1, Kounios and Holcomb (1992) replicated the Fischler et al. (1983) finding that predicate N400 is larger when the subject and predicate terms are comparatively unrelated. Furthermore, they found that the manipulation of sentence quantifier (hence sentence truth value) had no effect on predicate $\mathrm{N} 400$, also reminiscent of the absence of an effect of sentence negation obtained by Fischler et al. There was, however, an additional and surprising finding; namely, that the hierarchical level of the predicate term also had an effect on N400 amplitude: Exemplars yielded larger N400s than did categories. Furthermore, this level effect was apparently $a d-$ ditive with respect to the subject/predicate relatedness effect (Figure 2). A similar level effect was also obtained on the N400 to the subject term. Experiment 2 confirmed the existence of this hierarchical level effect in a task requiring subjects to make concrete/abstract judgments to single words; not only was the N400 level effect replicated, but it apparently had an additive relationship with respect to the effect of stimulus repetition. In addition, there were no significant effects on the peak latency of N400 in either experiment, further supporting the notion that upstream processes in a semantic verification experiment vary little, if at all, in duration.

These findings were especially surprising when considered in relation to the verification RTs obtained in Experiment 1 . Though there were no significant interactions among the effects of the three semantic variables on N400 amplitude, these same variables yielded strongly interacting effects on mean verification RT (see also Holcomb \& Kounios, 1990). 

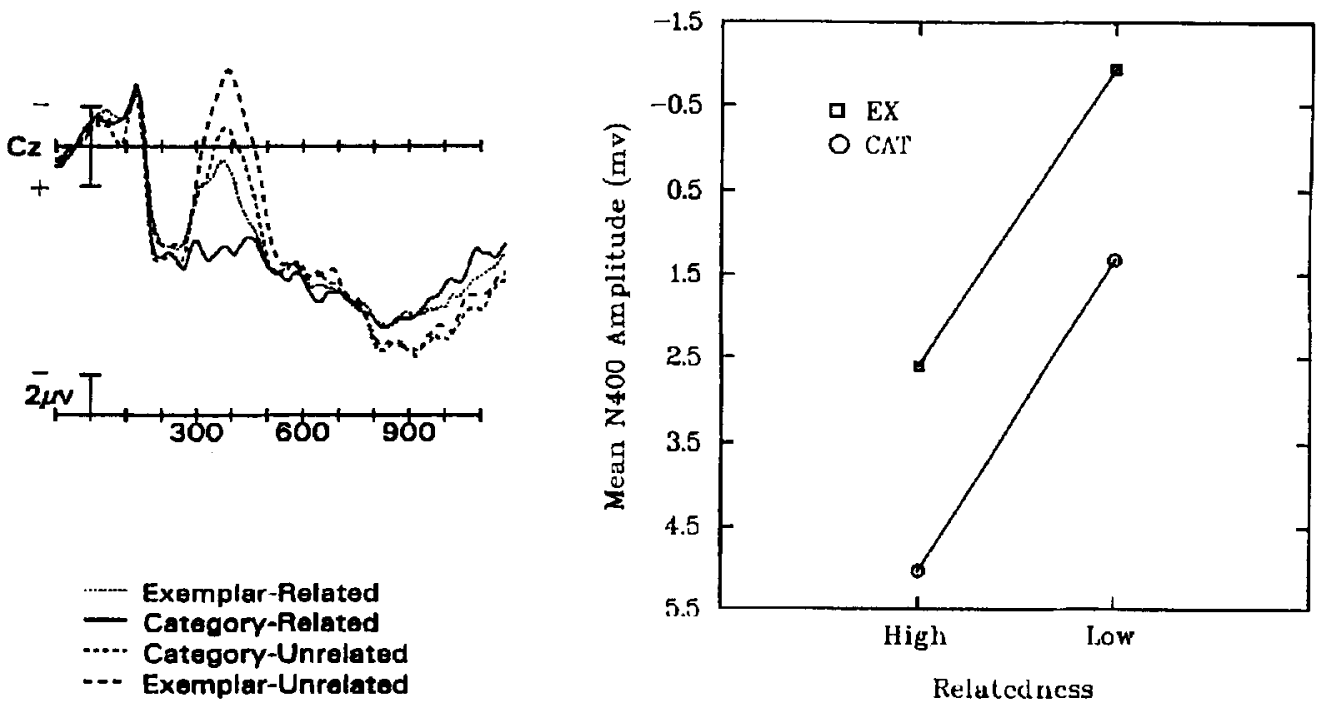

Figure 2. Left panel: Predicate event-related potentials at the $\mathrm{Cz}$ (i.e., central-midline) electrode site for four experimental conditions (relatedness $\times$ hierarchical level). Stímulus (i.e., predicate) onset is represented by the vertical calibration bar; horizontal axis represents time in milliseconds. Negative amplitude (microvolts) is plotted up on vertical axis. Right panel: Mean $\mathbf{N 4 0 0}$ amplitudes for midline electrode sites (Fz, frontal; $\mathbf{C z}$, central; Pz, parietal) as a function of semantic relatedness and hierarchical level (EX, exemplar; CAT, category). Both panels are from "Structure and Process in Semantic Memory: Evidence From Event-Related Brain Potentials and Reaction Times," by J. Kounios and P. J. Holcomb, 1992, Journal of Experimental Psychology: General, 121, pp. 468-469. Copyright 1992 by the American Psychological Association. Reprinted with permission.

Kounios and Holcomb (1992) interpreted the overall pattern of results as follows: (1) Since relatively early components such as the N400 (i.e., early with respect to RT) did not discernibly vary in latency, RT differences are likely attributable to effects on the duration of one or more late decision processes (assuming, of course, that the N400 is not a vestigial offshoot of the stream of processing leading up to the response, and that there are no relevant early processes that vary in latency that are not detectable by the ERP technique). This view is also consistent with the results of the additive-factors, SAT, and SAD studies reviewed earlier. (2) In contrast to the interactions exhibited by the RT data, the additive effects of the semantic variables on predicate $\mathrm{N} 400$ amplitude suggest that this component may reflect aspects of semantic memory structure, because N400 may (directly or indirectly) reflect predecision semantic access or retrieval mechanisms that reveal characteristics of the knowledge structure(s) within which they operate. In particular, (3) the additive effects on predicate $\mathrm{N} 400$ of the subject-predicate relatedness and predicate hierarchical-level factors suggest that these factors may represent orthogonal dimensions of semantic memory structure, the relatedness dimension being relational (i.e., a property of pairs of words) and the level dimension being nonrelational (i.e., a property of individual words). And lastly, (4) this semantic memory structure is also nonpropositional, because propositions possess truth value, while there were no effects of truth value (i.e., quantifier) on N400 amplitude. Kounios and Holcomb (1992) therefore argued that the semantic memory tapped in the classic verification paradigm stores single-word meanings and unquantified relations between concepts. Downstream processes must construct propositions for verification on the basis of this retrieved information.

As explained below, subsequent studies call for a partial revision and elaboration of this overall interpretation. These subsequent experiments were part of a separate line of ERP research that was later discovered to bear an unexpected relationship to the Kounios and Holcomb (1992) sentence verification study.

\section{Concreteness Effects}

An area of research that has rarely made theoretical contact with traditional semantic memory research (but see Glass, Millen, Beck, \& Eddy, 1985) concerns concreteness effects on semantic processing. The standard finding (see Paivio, 1990, 1991, and Schwanenflugel, 1991, for recent reviews) is that people process concrete words and sentences (e.g., apple, or "He bit into the juicy, red apple") more quickly and accurately than abstract ones (e.g., liberty, or "Liberty is the highest ideal of a civilized society"). Two main theories address this phenomenon.

Dual-coding theory. Dual-coding theory (Paivio, 1990) states that there are two knowledge representation systems: a verbal system that contains abstract word meanings and a concrete system that contains imagebased information. These two systems are hypothesized to be located in the left and right hemispheres of the brain, respectively. According to dual-coding theory, concrete words and sentences are processed more quickly and accurately because they are processed by both systems, since concrete words have both abstract meanings 
and associated imagery; in contrast, abstract words are processed only by the verbal system.

Context availability. The context-availability model (at least in its strong form, see Schwanenflugel, 1991; Schwanenflugel \& Shoben, 1983) rejects the notion of multiple types of representations or representational systems. Instead, it explains concreteness effects in terms of available inter- or intraitem context to support comprehension (Kieras, 1978). According to this notion, abstract words and sentences are simply more vague than concrete ones, containing less information and/or fewer strong connections to related material in semantic memory. This difference in amount of information or number of strong connections is thought to be quantitative and not qualitative (Schwanenflugel \& Shoben, 1983).

Testing the structural assumptions of dual-coding theory. A recent ERP study by Kounios and Holcomb (1994) tested the conflicting structural predictions of these two models. Dual-coding theory posits two independent (but interconnected) semantic memory structures (verbal and imaginal) located in the left and right hemispheres of the brain, respectively. On the other hand, the contextavailability model explicitly argues against such structure. Consequently, Kounios and Holcomb sought evidence of appropriate hemispheric lateralization of ERPs during the processing of abstract and concrete words. If these two types of words yield different topographic distributions of ERPs across scalp electrode sites consistent with the structural underpinnings of dual-coding theory, then the antistructure account of concreteness effects provided by the context-availability model would have to be revised or rejected. On the other hand, the absence of a meaningful difference in the topographic distributions of the ERPS would weigh against dual-coding theory. ${ }^{7}$

One of the tasks Kounios and Holcomb (1994) employed required subjects to view individual words and judge whether they were concrete or abstract. Figure 3 shows the average ERP waveforms (at 13 electrode sites) corresponding to the first presentations of concrete and abstract words. The top of the figure corresponds to the front of the head, the bottom of the figure to the back of the head, the right side of the figure to the right side of the head, and the left side of the figure to the left side of the head. Negative amplitude is plotted up (according to convention). Stimulus onset is shown by the vertical calibration bar at the bottom of the figure.

Since such waveforms may be difficult for the untrained eye to read, and for the sake of simplicity, it may be helpful to consider Figure 3 in relation to a quantification of certain aspects of these waveforms. The bot-

Concrete/Abstrect Task
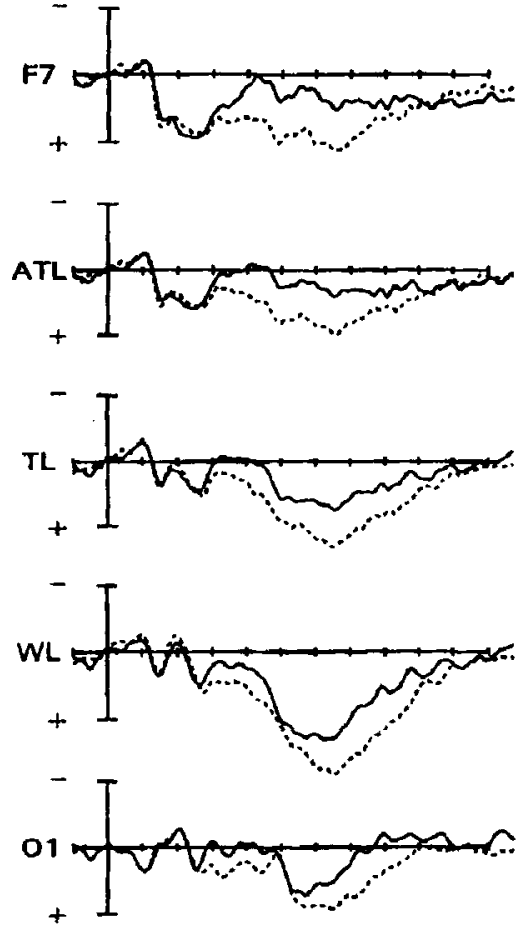

Concrete Words
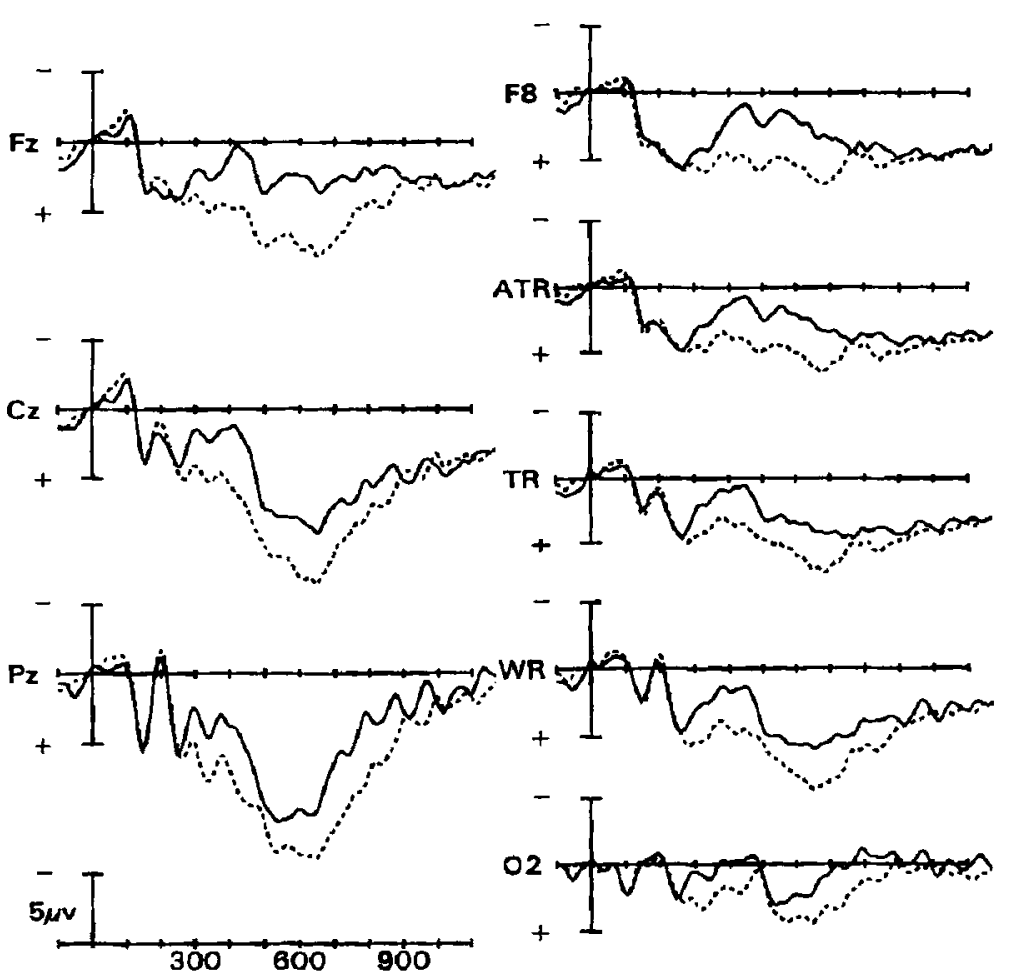

Abstract Words

Figure 3. Event-related potential waveforms for concrete/abstract word classification study. From "Concreteness Effects in Semantic Processing: ERP Evidence Supporting Dual-Coding Theory," by J. Kounios and P. J. Holcomb, 1994, Journal of Experimental Psychology: Learning, Memory, \& Cognition, 20, p. 818. Copyright 1994 by the American Psychological Association. Reprinted with permission. 


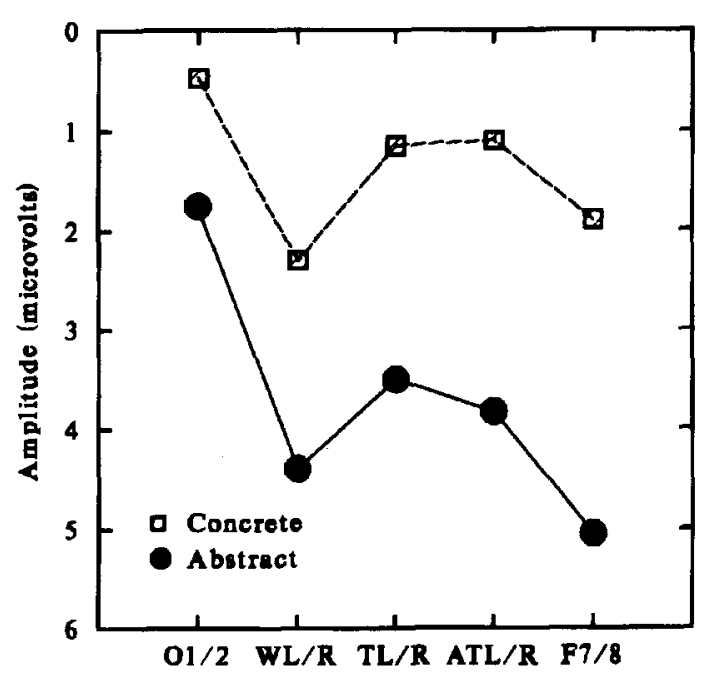

Electrode Site (posterior to anterior)

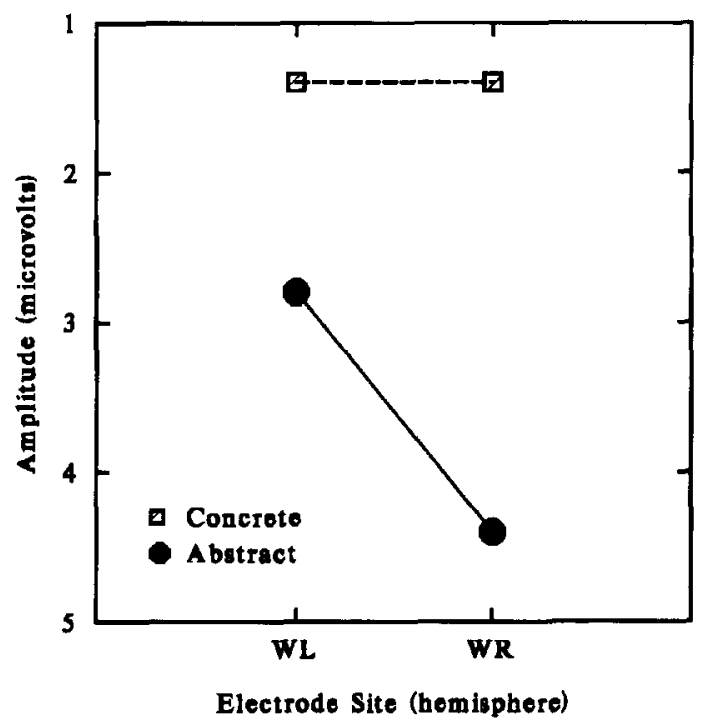

Figure 4. Quantified event-related potential results from concrete/abstract word classification study. Top panel: mean $\mathbf{N 4 0 0}$ to concrete and abstract words (vertical axis) from posterior to anterior electrode sites (horizontal axis: occipital, Wernicke's, temporal, anterior-temporal, frontal). Bottom panel: Mean N400 amplitude (microvolts) to concrete and abstract words (vertical axis) measured at Wernicke's left and right electrode sites (horizontal axis). From "Concreteness Effects in Semantic Processing," by J. Kounios and P. J. Holcomb, 1994, Journal of Experimental Psychology: Learning, Memory, \& Cognition, 20, p. 820. Copyright 1994 by the American Psychological Association. Reprinted with permission.

tom panel of Figure 4 shows the mean N400 amplitudes (i.e., the means for the time-window from 300 to $500 \mathrm{msec}$ ) for concrete and abstract words plotted as a function of hemisphere (specifically, the Wernicke's area electrode site over the left hemisphere and the homologous site over the right hemisphere). As the figure shows, N400 amplitude for concrete words was virtually identical across hemispheres, while N400 for ab- stract words was considerably greater over the left hemisphere than the right. This is exactly the pattern predicted by dual-coding theory: Concrete words are processed by both hemispheres, while abstract words are processed predominantly in the left. Furthermore, the top panel of the figure shows a different front/back topography; the difference in N400 amplitude between concrete and abstract words is greater at anterior than posterior electrode sites. Overall, these results provide firm support for the structural claims of dual-coding theory at the expense of the context-availability model.

ERPs and context availability. The hemispheric effects just described constitute strong evidence against a purely contextual account of concreteness effects. Nevertheless, it would be unrealistic to conclude from these results that context does not play any role in tasks used to study concreteness effects. Consequently, Holcomb, Kounios, and Anderson (1996) performed another ERP study (similar to Schwanenflugel \& Stowe's, 1989, RT study) to clarify the relative contributions of context and structure. This experiment involved the presentation of sentences, one word at a time, in which the concreteness of the sentence stems and final words was separately manipulated. Half of all the sentences were "congruent"; that is, they made sense (e.g., concrete: "The plumber could not fix the leak in the pipe without his tools"; abstract: "The total lack of knowledge on a topic is called ignorance"). The other half were "anomalous" (e.g., concrete sentence stem with concrete final word: "In order to bring the crops to market, the farmer had to hitch the horse up to the juice"; concrete stem with abstract final word: "Remember to bring the corkscrew so that we can open the bottle of vintage deduction"; abstract stem with abstract final word: "Convicted of a crime he didn't commit, the young man knew there was no such thing as foolish"; abstract stem with concrete final word: "Mad that his sister had blamed him, Jimmy said that it wasn't his potatoes"). As the examples show, each concrete sentence stem predicted a concrete final word, whether or not the actual final word rendered the sentence anomalous. Similarly, abstract sentence stems predicted abstract final words. Final-word predictabilities for concrete and $a b$ stract congruent sentences were equated by asking a separate group of subjects to produce best-completion final words for each sentence frame. The final group of sentences was selected so that the final-word production frequency was the same for concrete and abstract congruent sentences; final-word length and frequency were also equated. (Obviously, final-word predictability for concrete and abstract anomalous sentences could not be equated.) After each sentence's final word, the subject had to judge whether or not the sentence made sense and respond with the appropriate buttonpress.

To summarize, the main focus of the experimental design employed by Holcomb et al., 1996, was the final word of each sentence. Each final word was either concrete or abstract. Each sentence was (1) either congruent or anomalous and (2) predicted either a concrete or an abstract final word. Congruent sentences that were concrete 
always ended with a concrete word, and congruent abstract sentences always ended with an abstract word. Anomalous sentences predicted one type of word and could end in either type.

Figure 5 shows the ERPs to concrete and abstract final words for congruent sentences. The most important result for these conditions was that there were no statistically significant differences between these waveforms in any time-window examined (e.g., 150-300, 300-500, or $500-800 \mathrm{msec}$ ) or at any electrode site. Clearly the contextual support for each final word provided by the highly predictive sentence stem that preceded it overwhelmed any potential structural (e.g., hemispheric) effects of concreteness. In contrast, there were substantial and interesting differences for the anomalous sentences, as shown in Figure 6.

The left panel of Figure 6 quantifies the average ERP amplitudes for final words of anomalous sentences in the 150- to $300-\mathrm{msec}$ (i.e., P2) time-window preceding the 300 - to 500 -msec window typically used for N400 measurements (right panel). The graph shows the amplitudes for the four conditions of anomalous sentences: (1) concrete final word presented after a concrete context (i.e., after a concrete sentence stem), (2) concrete final word after an abstract context, (3) abstract final word after a concrete context, and (4) abstract final word after an abstract context. The most striking result revealed by this figure is the apparent additivity of amplitudes yielded by the factorial manipulation of final-word type and preceding context in the 150- to 300-msec time-window. In contrast, the right panel of Figure 6 shows the results for the same conditions in the subsequent time-window (viz., N400, 300$500 \mathrm{msec}$ ). Here there is a clear and unambiguous interaction between the word type and context factors.

A straightforward model can account for these results. For the anomalous sentences, the amplitude additivity that characterizes the 150 - to $300-\mathrm{msec}$ window suggests that word type and context are processed by independent and nonoverlapping populations of neurons during this timewindow (or a previous one). ${ }^{8}$ However, by the next latency window (N400), this independence clearly disappears; the N400 data cannot support the notion that these two types of information are processed independently. This suggests that for anomalous sentences, stable (i.e., structural) word type information and stem type (i.e., contextual) information are initially processed independently, followed by an attempt to integrate these two types of information.

In contrast, final words of congruent sentences showed no reliable word type effects during any time-window. This result might, at first, seem to weigh against dual-coding theory because one might expect concreteness effects to be evident in this experimental condition. However, the reason such effects were not found here is likely due to the nature of the technique employed. ERPs reflect the
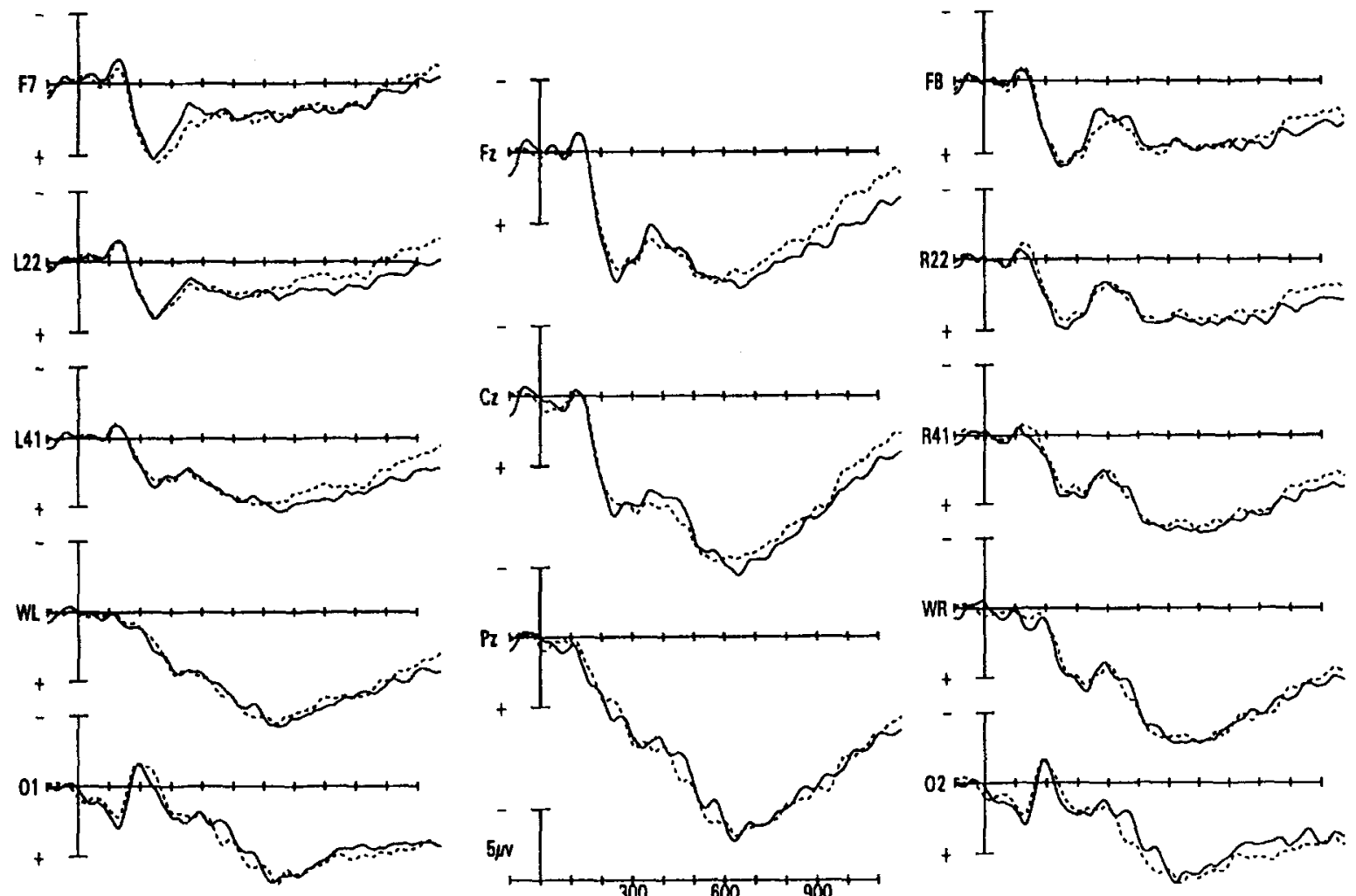

Figure 5. Event-related potential (ERP) waveforms for abstract and concrete final words of abstract and concrete congruent sentences (from Holcomb et al., 1996). Concrete ERPs are solid waves; abstract ERPs are dashed waves. See text for explanation. 

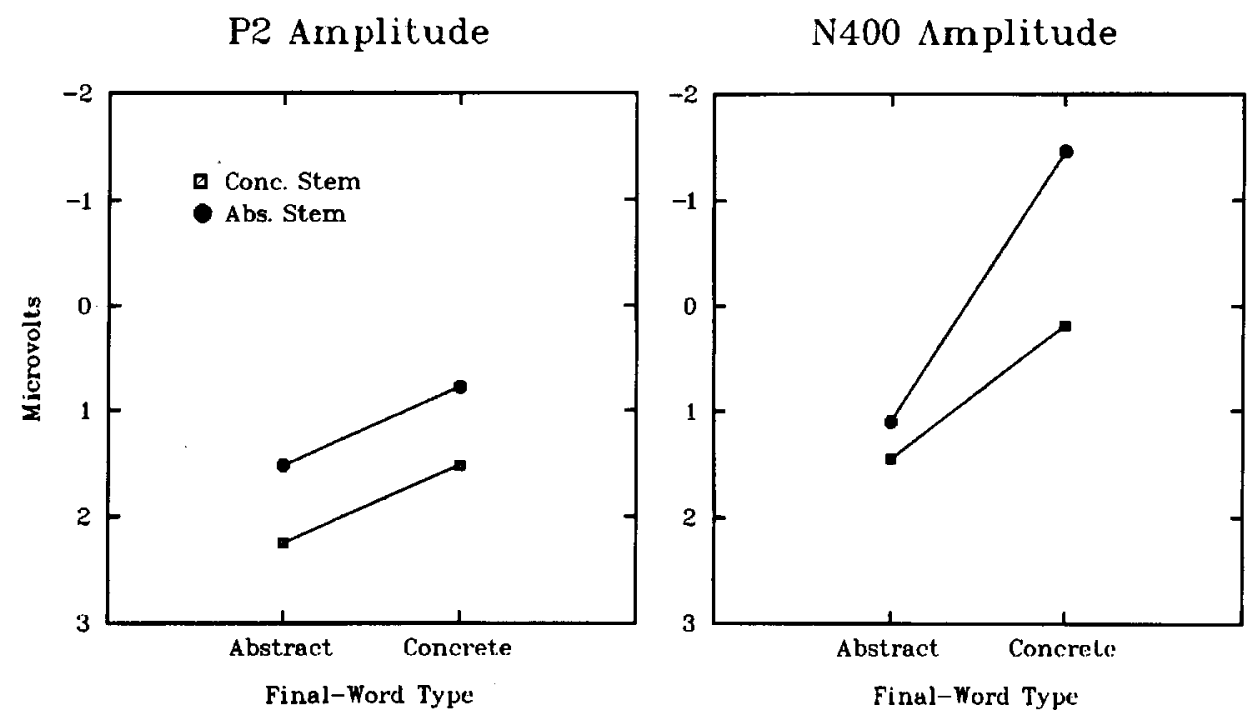

Figure 6. Anomalous-sentence results from Holcomb et al. (1996). Left panel: mean ERP amplitudes (microvolts) for abstract and concrete final words from anomalous sentences in the 150- to 300-msec timewindow. Right panel: corresponding results for $\mathbf{N} 400$ time-window (300-500 $\mathrm{msec})$.

brain's electrical response to discrete stimulus (or response) events; ongoing activity that is not significantly altered by or correlated with such an event is washed out by averaging the waveforms across individual trials. The use of sentence frames that were highly predictive of the meanings of the final words probably allowed subjects to accurately anticipate these meanings. If the final-word meaning were strongly anticipated or primed, then its subsequent presentation might not have caused enough of a change in the state of the brain's semantic representation system to result in an appropriate semantic effect on the ERP. In this case, one would not expect to detect final-word concreteness effects. ${ }^{9}$

Conclusions. The concreteness ERP studies reviewed above point to several important conclusions regarding semantic memory. First, the topographic effects found in the single-word study reported by Kounios and Holcomb (1994) show the importance of structure, in this case, theoretical structure corresponding to or derived from gross anatomical structure. Second, the final-word ERPs for concrete and abstract congruent sentences (Holcomb et al., 1996) suggest that a strongly supportive context can obfuscate the electrophysiological manifestations of this structure. Third, when the context is not congruent with the final word, word type and context effects are initially independent followed by a subsequent interaction. These results, together with those of the sentence verification study by Kounios and Holcomb (1992), suggest an interpretation of the cognitive processes that correspond to the $\mathrm{N} 400$. This interpretation is outlined below, along with its general significance for the study of semantic memory structure.

\section{N400 and Semantic Memory}

The N400 effects reviewed above can be understood as manifestations of one or more processes that attempt to combine information from different sources into a higher level (i.e., postlexical) concept representation suitable for further processing (see Osterhout \& Holcomb, 1995). This is most clearly illustrated by the sentence concreteness/context experiment just described (Holcomb et al., 1996). For anomalous sentences, context and word type are processed separately and independently during (or prior to) the 150 - to 300 -msec time-window; this amplitude additivity suggests that independent populations of neurons are responsible for processing these two types of information, with each neuronal population contributing independently to the overall amplitude of this component (but see note 8). This view makes particular sense given that the context preceding the anomalous final word strongly predicts some other (unrelated) best completion. It may be that words with little or no overlap in meaning are represented in populations of neurons that do not overlap. Consequently, the additive effects of context and word type on ERP amplitude in the 150- to 300 -msec time-window may signify a nonoverlap of neuronal populations representing two unrelated words: the explicit final word and the one implied or predicted by the context.

On the other hand, for anomalous sentences, context and word type interact strongly during the N400 timewindow, suggesting that here context and word type information are being processed by overlapping population(s) of neurons. Thus, N400 is plausibly interpreted as marking the attempted integration of two previously separate knowledge representations into a single representation. In this case, the hypothesized integration is, of course, unsuccessful, because the anomalous nature of these sentences does not permit meaningful integration. This contrasts with the case of the congruent sentences, which show no concreteness effects for either the 150 - to $300-$ or 300- to 500-msec time-windows. As noted, the 
absence of observable ERP concreteness effects suggests a virtually complete overlapping of neuronal populations corresponding to the meanings of the predicted and subsequently presented words.

How can this view be reconciled with the additive effects of subject-predicate relatedness and hierarchical level on predicate $\mathrm{N} 400$ obtained in the Kounios and Holcomb (1992) sentence verification study? They argued that this phenomenon was consistent with the notion that relatedness and hierarchical level are independent dimensions of semantic memory structure. However, the results of the Holcomb et al. (1996) concreteness/context study suggest a revision of this interpretation. Instead of reflecting a structural factor, relatedness may instead be construed as a contextual manipulation (i.e., with the sentence subject providing a context for the subsequent predicate). According to this scenario, the Kounios and Holcomb sentence verification results do not necessarily mean that the relatedness manipulation reflects only structural aspects of semantic memory, but that it may be, at least partially, a contextual factor. This view is consistent with the finding that effects on N400 amplitude of semantic priming interact with a clearly contextual variable such as repetition (e.g., Schwartz, Holcomb, \& Kounios, 1990), while the noncontextual hierarchical effect found in the single-word experiment of Kounios and Holcomb (1992, Experiment 2) did not interact with repetition. So, these sentence verification results may also be interpreted as supporting the independence of contextual and structural effects on upstream processes. However, in this case, the hypothesized independence of these two types of information survived the integration process presumably reflected by $\mathrm{N} 400$, hence the additive $\mathrm{N} 400$ effects. This suggests that integration may sometimes amount to linearly combining semantic representations and outputting a relatively simple function of the inputs. In the case of interacting $\mathrm{N} 400 \mathrm{~s}$, (attempted) integration that is either of another form or that uses different types of information as inputs can instead be nonlinear, resulting in a representation with characteristics that may be very different from the inputs to this process. However, the integration hypothesis clearly suggests caution in generalizing across experimental tasks, since the type of integration occurring may very well depend upon characteristics of the task (Bentin \& Peled, 1990; Holcomb, 1988). Nevertheless, systematic manipulation of semantic and contextual variables may, in the future, yield a classification of concepts into linear and nonlinear types based on $\mathrm{N} 400$ and other ERP results.

However, N400 does not seem to represent the final step in the semantic integration process. Kounios and Holcomb (1992) found no effects of sentence quantifier on predicate $\mathrm{N} 400$. Since a sentence's quantifier determines its truth value (e.g., "All dogs are animals" vs. "No dogs are animals"), the absence of an effect of quantifier on predicate $\mathrm{N} 400$ suggests that this integration process did not (in this task) incorporate truth value into its outputted representation; hence it is nonpropositional. Subsequent mechanisms are likely responsible for constructing the final propositional representation.

\section{A FRAMEWORK FOR UNDERSTANDING SEMANTIC MEMORY STRUCTURE}

Each of the RT, SAT, SAD, and ERP studies discussed above reveal something about semantic memory structure. Unfortunately, the term structure has been used somewhat equivocally here (and elsewhere). Semantic memory can actually be described as exhibiting at least three levels of structure, referred to below as micro, macro, and global. Different kinds of experimental results elucidate different levels of this overall structure. Each of these levels will undoubtedly prove important to future semantic memory research, as each makes use of some unique theoretical primitives and different research methods.

\section{Overview}

The notion of a knowledge representation is obviously an abstraction. Nevertheless, a knowledge representation must, by definition, be accomplished in (or at least reside in) a physical medium, in this case, the brain. The physical nature of the brain, in turn, imposes certain constraints on the abstract characteristics of a representation. An analysis of these constraints is useful in that it provides a concrete perspective on the otherwise ephemeral quality of a representation.

The analysis begins with the metaphor of a representation as a physical "thing." At the first (micro) level, a thing must be "made of" something. For this purpose, most representational frameworks make use of the notion of units of knowledge, usually features or attributes, as a theoretical primitive. Whatever "units" are (e.g., corresponding to individual neurons or to populations of neurons), they can be characterized by the qualitative distinction of type and the quantitative distinction of informational grain size. At the second (macro) level, a set of units (corresponding to a neuronal population) can collectively comprise or instantiate a representation. Such a set of units also has grain size, which is a function of the number of units in the set as well as the grain size of the individual units. A set of units also has an internal organization specifying the relations among the units; this organization can be described in both structural (e.g., cytoarchitectonic) and dynamic (e.g., neurophysiological) terms. At the third (global) level, a representation has location (e.g., neuroanatomical) and density (e.g., spatially distributed or localized). These three levels are discussed in more detail below and are characterized in Table 1.

\section{Microstructure}

As just described, the term microstructure refers to characteristics of the representational medium, namely, to the grain size and type of theoretical units constituting a semantic memory representation. ${ }^{10}$ Here, the grain size distinction refers to the amount of information represented by each unit. Unit grain size is therefore related 
Table 1

Levels of Semantic Memory Structure

\begin{tabular}{lccc}
\hline \multicolumn{3}{c}{ Characteristics of the Levels } \\
\cline { 2 - 4 } Level & $\begin{array}{c}\text { Different } \\
\text { Representational } \\
\text { Media }\end{array}$ & $\begin{array}{c}\text { Disjoint } \\
\text { Neuronal } \\
\text { Populations }\end{array}$ & $\begin{array}{c}\text { Separate } \\
\text { Anatomical } \\
\text { Structures }\end{array}$ \\
\hline Micro & ++ & $?$ & $?$ \\
Macro & 0 & 0 & 0 \\
Global & + & + & + \\
\hline
\end{tabular}

Note-- ++, a distinction or relationship by definition or logical implication; + , a probable relationship; ?, it is unknown whether such a relationship exists; 0 , the dimensions in question are probably independent.

to the granularity of the overall representation; that is, where it lies on the discrete - continuous dimension, because a representation composed of larger grains or chunks lies closer to the discrete end of this continuum (i.e., if the number of units is held constant). The unit-type distinction refers to the possibility that representations are decomposable into qualitatively different types of primitive units (e.g., units that are perceptual or abstract in nature). The relationship, if any, between the unit type and grain size distinctions is presently unknown.

Table 1 describes some characteristics of the three levels of structure. Each cell of the table indicates the relationship between a level of representational structure (i.e., micro, macro, and global, given by the rows) and certain characteristics that are applicable to the levels (given by the columns). More specifically, each cell notes whether a difference between two representations at a given level of structure signifies a difference between these representations in their representational media, differences in the neuronal populations that instantiate these representations, or whether entirely different neuroanatomical structures are responsible for instantiating the two representations.

The left-most column refers to differences between representations due to differences in the representational medium. The two plus signs in the upper left-hand cell indicate that this characteristic defines the microstructural level, in other words, that unit grain size and type define representational microstructure.

The cell in the second column of the first row of Table 1 refers to whether a difference at the microstructural level between two representations implies that these representations involve disjoint populations of neurons (i.e., within a particular anatomical brain structure; see below). In other words, must two concepts (or different components of the meaning of a single concept; see Damasio \& Damasio, 1992) that have a qualitative (i.e., unit type) difference in their representational media be represented by disjoint populations of neurons? (Clearly, the quantitative grain size distinction makes no such implication.) A question mark has been placed in this cell, since the answer to this question is presently unknown. For instance, if (given some mapping of neurons on to theoretical units; see note 10) a particular neuron can directly participate in the representation of concepts (or components of concepts) composed of only one type of theoretical unit (and perhaps only at a particular level of granularity), then differ- ences between representations in unit type (or unit grain size) may signify the degree of nonoverlap between the neuronal populations instantiating the representations.

The third column refers to whether the neuronal populations responsible for representating two concepts (or two components of the meaning of a single concept) that differ at the microstructural level must be located in separate anatomical brain structures (e.g., the two cerebral hemispheres or a pair of smaller structures). The question mark in the upper-right cell of Table 1 indicates that it is presently unknown whether a microstructural difference between two representations necessarily indicates that the representations are maintained in separate anatomical brain structures.

Instead of directly focusing on the issues of unit type and grain size, most semantic memory research has focused on the appropriate type(s) of representation to employ in theory construction (e.g., propositional, imaginal, relational, etc.). There seems to be good evidence for the existence of qualitatively different types of representations (Paivio, 1990; Shepard \& Cooper, 1982). Future research may show that a fundamental difference in type of representation (e.g., verbal vs. imaginal) always implies a corresponding anatomical distinction, as in Paivio's (1990) dual-coding theory. However, the microstructural distinction does not refer to whether two representations are qualitatively different, but to whether the fundamental primitive units constituting a representation are qualitatively different. It may be possible to construct qualitatively different types of representations from a single type of unit, in which case the existence of two qualitatively different types of representations does not necessarily imply nonoverlapping neuronal populations (or anatomical brain structures). However, it may be that different anatomical structures utilize fundamentally different types of theoretical units as representational building blocks.

There are several techniques for studying representational grain size (reviewed by Meyer et al., 1988; Miller, 1988). The grain size issue has been primarily addressed by SAD (Kounios et al., 1994; Kounios et al., 1987). On the other hand, as there are no experimental techniques specifically designed to investigate distinctions of unit type, the most profitable approach to this problem may be a purely theoretical one: Given a specific type of unit, is it theoretically possible to construct qualitatively different types of representations?

\section{Macrostructure}

Representational macrostructure is characterized by the size of a representation, with size here defined by the number and grain size of the units constituting the representation, and by the internal organization or relational structure of these units within the representation. Since there are currently no theoretical or empirical reasons to think that representational size or organization imply anything about representational medium, a zero has been placed in the "Different Representational Media" cell of the macrostructure row of Table $1 .{ }^{11}$ As for the "Separate 
Anatomical Structures" cell, there is also currently no reason to think that a given anatomical brain structure cannot form representations of different sizes or relational structures. Consequently, a zero has also been placed in this cell to indicate that differences in representational macrostructure probably imply nothing about neuroanatomy. Instead, the focus of the present discussion of macrostructure is primarily concerned with the issue of distinguishable neuronal populations (within a given anatomical structure).

Clearly, there is no a priori reason to think that two representations that differ in size or organization must be instantiated in disjoint neuronal populations; hence the zero in the middle cell of Table 1 . Overall, the middle row of the table suggests that differences in representational size and internal organization do not, to the best of our knowledge, imply anything about the nature of the representational media, the nonoverlap of neuronal populations that form the neural substrate of the representations, or the anatomical location and density (localized vs. distributed) of representations. However, in spite of the seeming uninformativeness of the macro level, there is much theoretical potential here, particularly with regard to the issue of disjoint neuronal populations.

The additive effects on ERP component amplitudes obtained in the concreteness/context study of Holcomb et al. (1996) and the sentence verification study of Kounios and Holcomb (1992) indicate that semantic representations could be associated with disjoint sets of neurons, with each set providing an independent contribution to the amplitude of an ERP component (see also Johnson, 1993; Schweickert, 1993). This, of course, weighs against any fully distributed model of semantic representation, because such models (by definition) assume that all of the units in a network participate fully in every representation (for a discussion, see Feldman, 1989; Hinton, McClelland, \& Rumelhart, 1986). The existence of disjoint populations of neurons processing different aspects of meaning therefore suggests representational modularity. (A modular representational scheme can, of course, take advantage of principles of distributed representation within each module.)

In addition, a modular form of representation can easily account for results such as the hierarchical level effect on N400 found by Kounios and Holcomb (1992). They found that exemplar terms yielded larger N400s than did category terms. If concepts are viewed in terms of sets of features, then a category term can be transformed into a (subordinate) exemplar term by adding features (i.e., increasing representational size; cf. Hinton et al., 1986). Similarly, an exemplar term can be transformed into a (superordinate) category concept by subtracting features. Given an appropriate mapping of features on to neurons, more neural activity (e.g., a larger N400 negativity resulting from the firing of a greater number of neurons) would be expected during processing of exemplar terms than during processing of category terms.

One research strategy for investigating semantic memory macrostructure has already been mentioned. Evidence of additive effects of semantic variables on ERP component amplitudes suggests modularity of neural representation. Hence, future factorial ERP experiments along the lines of those reviewed above should yield valuable evidence concerning semantic memory organization. There is, however, one caveat to this approach. Since the conclusion that two factors yield additive effects is dependent on the acceptance of the null hypothesis (i.e., no interaction), researchers must take pains to hypothesize additivity based only on very sensitive statistical tests.

A complementary approach to analyzing ERP additivity has been discussed by Johnson (see Johnson, 1993, for a review). He pointed out that if two factors produce independent effects on the amplitude of an ERP component, then these independent contributions are probably produced by different populations of neurons (i.e., neural "generators"; however, see note 8). Obviously, two disjoint networks of neurons are likely to (but do not necessarily) occupy different physical regions of the brain, so these independent contributions to component amplitude are likely to be generated in different locations in the brain. This would result in different topographic distributions of the effects of the two factors across the various electrode sites on the scalp (i.e., after the amplitudes have been appropriately normalized; see McCarthy \& Wood, 1985). For example, semantic factors A and B might yield additive effects on N400 amplitude (i.e., a nonsignificant $\mathrm{A} \times \mathrm{B}$ interaction). However, if these (additive) effects originate in different areas of the brain, then the specific additive relationship between $A$ and $B$ should vary across electrode sites.

This approach will undoubtedly prove extremely useful, although another caveat is in order. The resolution of topographic ERP maps necessarily depends on the type, size, spatial orientation, and depth of neural generators, as well as on the number and distribution of electrodes over the scalp (Duffy, Iyer, \& Surwillo, 1989; Nunez, 1990; Wong, 1991). Even under the best of circumstances, surface electrode recordings may not always yield evidence of differential scalp topography. For instance, the more deeply situated within the brain is a generator, the greater the outward spread (i.e., volume conduction) of the electrical field emanating from the generator. This is analogous to a flashlight beam growing wider with distance. Alternately, the generation of a specific ERP component may not occur in a relatively small, well-defined region of neural tissue; the component may be produced by a large, spatially diffuse collection of neurons. In this case, distinct ERP topographies may not be easily discriminated by conventional methods.

It should be kept in mind, however, that additive amplitude effects do not necessarily directly result from separate contributions from two or more independent neural generators. As already discussed, such a pattern could also occur if a single neural generator is producing the ERP component in question, and if that neural generator yields outputs that are a linear combination of its inputs (see note 8 ). So, finding additive amplitudes in the absence of differential topographies may suggest that 
a single generator is responsible for producing the additivity. This conclusion would gain added credibility if the single topographic pattern were fairly distinct and detailed, indicating a degree of spatial resolution high enough to allow one to detect subtle topographic differences resulting from multiple neural generators.

The notions of additivity discussed above, whether resulting from a single or from multiple neural generators, suggest an interesting approach to studying semantic memory structure, semantic context, and the nature of concepts. First, consider the case in which one neural generator yields outputs reflecting a linear combination of inputs from two nonoverlapping neuronal populations influenced by two distinct classes of semantic variables, structural and contextual:

$$
A=[C+S]+Z \text {. }
$$

In this equation, $A$ is the measured amplitude of the component in question, $C$ is the contribution to this amplitude from a single generator corresponding to a neuronal population representing contextual information, $S$ is the contribution from a neuronal population representing stable (i.e., structural) semantic information, and $Z$ is the contribution from all other sources. This is the simplest conceptualization, and can be analyzed further. For instance, it is unlikely that semantic memory structure is organized along only one dimension; there are a number of semantic variables that may reflect real cognitive and neural distinctions. If these semantic factors represent truly independent dimensions of semantic structure, then they should yield amplitude effects that are additive with respect to each other and with respect to the effects of contextual manipulation:

$$
A=\left[C+\left(s_{1}+s_{2}+\ldots+s_{n}\right)\right]+Z .
$$

Such a result would argue in favor of a complex, decomposable semantic memory structure. The lack of interactions among the factors could, however, weaken the empirical distinction between contextual and structural factors, unless, for instance, the structural subfactors had a coherent set of topographic distributions that was qualitatively different from the topographic pattern due to contextual manipulations. Then it could be argued that the distinction between structure and context is more than purely theoretical. Structural semantic factors may, however, interact, suggesting that there may exist a stable semantic memory structure that is independent of the influence of context, but that this structure cannot be characterized by independent dimensions, for example,

$$
A=\left[C+\left(s_{1} \times s_{2} \times \ldots \times s_{n}\right)\right]+Z
$$

(where multiplication represents just one possible form of interaction). Appropriate experimentation may also reveal some combination of additive and interacting semantic factors.

Similarly, contextual influence on semantic representation may not be monolithic. It may consist of subfactors that are independent:

$$
A=\left[\left(c_{1}+c_{2}+\ldots+c_{n}\right)+S\right]+Z ;
$$

or that interact:

$$
A=\left[\left(c_{1} \times c_{2} \times \ldots \times c_{n}\right)+S\right]+Z .
$$

The discovery of independent dimensions of semantic context would be just as significant as the discovery of independent dimensions of stable semantic structure. Such an analysis could provide invaluable evidence concerning the existence of separate neuronal populations processing different aspects of linguistic context during comprehension.

In summary, the methods of analyzing amplitude additivities and scalp topography may reveal whether semantic memory is composed of independent structural dimensions, and whether semantic context is monolithic or can also be decomposed.

\section{Global Structure}

The last row of Table 1 describes aspects of global structure, that is, differences between semantic representations corresponding to neuroanatomical distinctions. The best known theory relevant to this notion is probably the dualcoding theory of Paivio (1990), which posits lateralized representational systems for concrete and abstract meanings. Damasio and Damasio (1992) have also offered a number of hypotheses concerning the functional neuroanatomy of conceptual representation.

As indicated previously, at the global level, knowledge representations can be characterized by neuroanatomical location (i.e., the brain regions directly involved in sustaining or processing a representation) and density (i.e., distributed vs. localized). These dimensions are obviously not independent, as a fully distributed representation could, in principle, involve a very large portion of the brain and could therefore not easily be analyzed in terms of neuroanatomical location.

Do differences between representations in location or density imply that separate anatomical structures are responsible for different representations? If so, this would imply a degree of modularity inconsistent with fully distributed representation. However, full distribution does not seem to be theoretically practical (Hinton et al., 1986) or neuropsychologically plausible (Saffran \& Schwartz, 1994). If at least a weak form of localizationism is granted, then the question of the involvement of separate anatomical structures must be given at least a qualified "yes," hence the single plus sign in the third column of the third row of Table 1. For the same reason, the second column of the third row receives a single plus sign, indicating the likelihood of disjoint neuronal populations given a global level difference between representations (because differences in location do not necessarily preclude overlap of neuronal populations). And as for the first column, the question of whether differences in representational location or density indicate different representational media must also receive a tentative "yes" (i.e., a plus sign) if only because distributed representations make use of units of smaller grain size than do localized (i.e., symbolic) 
representations. However, it also seems likely that differences in location are associated with differences in representational media (Paivio, 1990), although this depends in part on whether qualitatively different types of representations (e.g., abstract and concrete) must be constructed from different sorts of representational units, or whether a common type of primitive unit will suffice.

The significance of the distinction between the global and macro levels may reflect degree of structural stability of knowledge representation. For instance, the participation of distinct anatomical brain regions in semantic representation indicates a structural segmentation between populations of neurons. On the other hand, the operation of distinct neural populations within a given anatomical structure may not reflect the same degree of representational stability at the microstructural level. The representation of two concepts within a given anatomical region may result in the "segmentation" of this region into independent neuronal populations. However, the geometrical instantiation of this segmentation may be highly abstract: Different collections of specific neurons within this region may be involved each time the same concepts are activated. This might signify that a concept is being represented by different, though, in an abstract sense, equivalent sets of units each time it is activated. But if some of these otherwise equivalent sets possess different connections to other representations, then the activation of a particular concept on different occasions could result in differential contextual influence.

As for research strategies appropriate for the study of global structure, any method that examines the underlying functional neuroanatomy is a good candidate. For example, ERPs can be useful if the researcher goes beyond quantifying the topographic distributions of components and utilizes available methods for inferring the number and locations of neural generators, possibly in concert with magnetoencephalography (Regan, 1989; Wong, 1991). This may complement neuropsychological (Saffran \& Schwartz, 1994) and functional neuroimaging studies (Posner \& Raichle, 1994).

\section{CONCLUSION}

This review has hopefully made five points: (1) Though the early RT studies of semantic memory offered a glimpse of its structure, that approach was not able to yield rich enough data to reject competing nonstructural accounts of the results. (2) Behavioral and electrophysiological timecourse measures offer greater insight into the processing architecture that underlies semantic verification. Knowledge of this processing architecture permits inferences about the nature of the information being processed. (3) Electrophysiological methods are particularly useful in this regard, because they offer extremely fine-grain temporal evidence and useful (but less precise) neuroanatomical evidence, aspects of which are not influenced by ad hoc decision mechanisms. (4) The topographic and amplitude-additivity data from such ERP studies suggest considerable structure. (5) A theoretical analysis of the var- ious possible levels of structure that takes into consideration these findings as well as relevant physical properties of the brain suggests further avenues and methods of investigation.

The notion of stable semantic structure has been unpopular in recent years, and not without some justification, since the importance of context to human cognition cannot be overemphasized. Nevertheless, it is also important to recognize that semantic memory, because it is realized in a structured brain, must itself exhibit structure; moreover, this structure can be analyzed on more than one level. Cognitive psychologists and neuroscientists are beginning to take advantage of the variety of research techniques suitable for studying these levels. In particular, electrophysiological methods offer much promise as a tool for exploring both the structural and contextual bases of knowledge representation.

\section{REFERENCES}

ANDERSON, J. R. (1978). Arguments concerning representations for mental imagery. Psychological Review, 85, 249-277.

ANDERSON, J. R. (1983). The architecture of cognition. Cambridge, MA: Harvard University Press.

BARSALOU, L. W. (1987). The instability of graded structure: Implications for the nature of concepts. In U. Neisser (Ed.), Concepts and conceptual development: Ecological and intellectual factors in categorization (pp. 101-140). Cambridge: Cambridge University Press.

BattiG, W. F., \& Montague, W. E. (1969). Category norms of verbal items in 56 categories: A replication and extension of the Connecticut category norms. Journal of Experimental Psychology Monographs, 80 (3, Pt. 2)

BENTIN, S., \& PELED, B.-S. (1990). The contribution of task-related factors to ERP repetition effects at short and long lags. Memory \& Cognition, 18, 359-366.

BroadBent, D. E. (1958). Perception and communication. Elmsford, NY: Pergamon.

Brown, C., \& HagoorT, P. (1993). The processing nature of the N400: Evidence from masked priming. Journal of Cognitive Neuroscience, 5, 34-44.

CASEY, P. J. (1992). A reexamination of the roles of typicality and category dominance in verifying category membership. Journal of Experimental Psychology: Learning, Memory, \& Cognition, 18, 823-834.

CASEY, P. J., \& HEaTh, R. A. (1989). A semantic memory sentence verification model based on relative judgment theory. Memory \& Cognition, 17, 463-473.

Casey, P. J., \& Heath, R. A. (1990). Semantic memory retrieval: Deadlining the typicality effect. Quarterly Journal of Experimental Psychology, 42A, 649-673.

Chaffin, R., \& Herrmann, D. J. (1988), The nature of semantic relations: A comparison of two approaches. In M. Evens (Ed.), Relational models of the lexicon (pp. 289-334). New York: Cambridge University Press.

CHANG, T. M. (1986). Semantic memory: Facts and models. Psychological Bulletin, 99, 199-220.

Chumbley, J. I. (1986). The roles of typicality, instance dominance, and category dominance in verifying category membership. Journal of $E x$ perimental Psychology: Learning, Memory, \& Cognition, 12, 257-267.

Collins, A. M., \& LofTus, E. F. (1975). A spreading activation theory of semantic processing. Psychological Review, 82, 407-428.

Collins, A. M., \& Quillian, M. R. (1969). Retrieval time from semantic memory. Journal of Verbal Learning \& Verbal Behavior, 8, 240-247.

Corbett, A. T., \& Wickelgren, W. A. (1978). Semantic memory retrieval: Analysis by speed-accuracy tradeoff functions. Quarterly Journal of Experimental Psychology, 30, 1-15.

Damasio, A. R., \& Damasio, H. (1992). Brain and language. Scientific American, 267, 88-95.

DUFFY, F. H., IYER, V. G., \& SURWILlo, W. W. (1989). Clinical electro- 
encephalography topographic brain mapping: Technology and practice. New York: Springer-Verlag.

Feldman, J. A. (1989). Neural representation of conceptual knowledge. In L. Nadel, L. A. Cooper, P. Culicover, \& R. M. Harnish (Eds.), Neural connections, mental computation (pp. 69-103). Cambridge, MA: MIT Press.

Fischler, 1., Bloom, P. A., Childers, D. G., Roucos, S. E., \& Perry, N. W. (1983). Brain potentials related to stages of sentence verification. Psychophysiology, 20, 400-409.

FISCHLER, I., \& RANEY, G. E. (1991). Language by eye: Behavioral, autonomic and cortical approaches to reading. In J. R. Jennings \& M. G. H. Coles (Eds.), Handbook of cognitive psychology: Central and autonomic nervous system (pp. 511-574). New York: Wiley.

Glass, A. L., \& HolyoaK, K. J. (1975). Alternative conceptions of semantic memory. Cognition, 3, 313-339.

Glass, A. L., Millen, D. R., BecK, L. G., \& EdDy, J. L. (1985). Representation of images in sentence verification. Journal of Memory \& Language, 24, 442-465.

GRUENENFELDER, T. M. (1986). Relational similarity and context effects in category verification. Journal of Experimental Psychology: Learning, Memory, \& Cognition, 12, 587-599.

HAMPTON, J. A. (1984). The verification of property and category statements. Memory \& Cognition, 12, 345-354.

HillyaRd, S. A., \& PICTON, T. W. (1987). Electrophysiology of cognition. In F. Plum (Ed.), Handbook of physiology: Section 1. Neurophysiology (pp. 519-584). New York: American Physiological Society.

Hinton, G. E., MCClelland, J. L., \& Rumelhart, D. E. (1986). Distributed representations. In J. L. McClelland, D. E. Rumelhart, \& the PDP Research Group (Eds.), Parallel distributed processing: Explorations in the microstructures of cognition (Vol. 1, pp. 77-109). Cambridge, MA: MIT Press.

HolcomB, P. J. (1988). Automatic and attentional processing: An eventrelated brain potential analysis of semantic priming. Brain \& Language, 35, 66-85.

HoLCOMB, P. J. (1989). The effects of stimulus energy on language sensitive ERPs. Psychophysiology, 26, S33.

Holcomв, P. J. (1993). Semantic priming and stimulus degradation: Implications for the role of the N400 in language processing. Psychophysiology, 30, 47-61.

Holcomb, P. J., \& Kounios, J. (1990). Semantic memory: An ERP and reaction time analysis. In C. H. M. Brunia, A. W. K. Gaillard, \& A. Kok (Eds.), Psychophysiological brain research (pp. 285-288). Tilburg: Tilburg University Press.

Holcomb, P. J., Kounios, J., \& Anderson, J. E. (1996). Concreteness, context, and semantic processing: An ERP investigation. Manuscript in preparation.

HoLLAN, J. D. (1975). Features and semantic memory: Set-theoretic or network model? Psychological Review, 82, 154-155.

JoHNSON, R. (1993). On the neural generators of the P300 component of the event-related potential. Psychophysiology, 30, 90-97.

KIERAS, D. (1978). Beyond pictures and word: Alternative information processing models for imagery effects in verbal memory. Psychological Bulletin, 85, 532-554.

KinTsCH, W. (1980). Semantic memory: A tutorial. In R. Nickerson (Ed.), Attention and performance VIII (pp. 595-620). Hillsdale, NJ: Erlbaum.

Komatsu, L. K. (1992). Recent views of conceptual structure. Psychological Bulletin, 112, 500-526.

Kounios, J. (1993). Process complexity in semantic memory. Journal of Experimental Psychology: Learning, Memory, \& Cognition, 19 338-351.

Kounios, J., \& Holcomb, P. J. (1992). Structure and process in semantic memory: Evidence from event-related brain potentials and reaction times. Journal of Experimental Psychology: General, 121, 459-479.

KounIos, J., \& Ноцсомв, P. J. (1994). Concreteness effects in semantic processing: ERP evidence supporting dual-coding theory. Journal of Experimental Psychology: Learning, Memory, \& Cognition, 20, 804-823.

Kounios, J., Montgomery, E. C., \& Smith, R. W. (1994). Semantic memory and the granularity of semantic relations: Evidence from speed-accuracy decomposition. Memory \& Cognition, 22, 729-741.

Kounios, J., Osman, A. M., \& MEyer, D. E. (1987). Structure and pro- cess in semantic memory: New evidence based on speed-accuracy decomposition. Journal of Experimental Psychology: General, 116, 325 .

Kounios, J., \& Smith, R. W. (1995). Speed-accuracy decomposition yields a sudden insight into all-or-none information processing. Acta Psychologica, 90, 229-241.

KuTAS, M., \& Hillyard, S. A. (1980). Reading senseless sentences: Brain potentials reflect semantic incongruity. Science, 207, 203-205.

KuTAS, M., \& Hillyard, S. A. (1984). Brain potentials during reading reflect word expectancy and semantic association. Nature, 307, 161163.

Kutas, M., Lindamood, T., \& Hillyard, S. A. (1984). Word expectancy and event-related brain potentials during sentence processing. In S. Kornblum \& J. Requin (Eds.), Preparatory states and processes (pp. 217-238). Hillsdale, NJ: Erlbaum.

Larochelle, S., \& Pineau, H. (1994). Determinants of response times in the semantic verification task. Journal of Memory \& Language, 33, 796-823.

LORCH, R. (1982). Priming and search processes in semantic memory: A test of three models of spreading activation. Journal of Verbal Learning \& Verbal Behavior, 21, 468-492.

MCCARThy, G., \& Nobre, A. C. (1993). Modulation of semantic processing by spatial selective attention. Electroencephalography \& Clinical Neurophysiology, 88, 210-219.

McCarThy, G., \& Wood, C. C. (1985). Scalp distributions of eventrelated potentials: An ambiguity associated with analysis of variance models. Electroencephalography \& Clinical Neurophysiology, 62, 203-208.

MCClelland, J. L. (1979). On the time relations of mental processes: An examination of systems of processes in cascade. Psychological Review, 86, 287-330.

McCloskey, M. (1980). The stimulus familiarity problem in semantic memory research. Journal of Verbal Learning \& Verbal Behavior, 19, 485-502.

MCCloskey, M., \& GluCKSBERG, S. (1979). Decision processes in verifying category membership statements: Implications for models of semantic memory. Cognitive Psychology, 3, 313-339.

Mervis, C. B., Catlin, J., \& Rosch, E. (1976). Relationships among goodness-of-example, category norms, and word frequency. Bulletin of the Psychonomic Society, 7, 283-284.

MEYER, D. E. (1970). On the representation and retrieval of stored semantic information. Cognitive Psychology, 1, 242-299.

Meyer, D. E., Irwin, D. E., Osman, A. M., \& Kounios, J. (1988). The dynamics of cognition and action: Mental processes inferred from speed-accuracy decomposition. Psychological Review, 95, 183-237.

MilleR, J. (1988). Discrete and continuous models of human information processing: Theoretical distinctions and empirical results. Acta Psychologica, 67, 191-257.

NEELY, J. H. (1991). Semantic priming effects in visual word recognition: A selective review of current findings and theories. In D. Besner \& G. Humphreys (Eds.), Basic processes in reading: Visual word recognition (pp. 264-336). Hillsdale, NJ: Erlbaum.

NewelL, A. (1973). You can't play 20 questions with nature and win: Projective comments on the papers in this symposium. In W. G. Chase (Ed.), Visual information processing (pp. 283-308). New York: Academic Press.

NunEz, P. L. (1990). Physical principles and neurophysiological mechanisms underlying event-related potentials. In J. W. Rohrbaugh, R. Parasuraman, \& R. Johnson, Jr. (Eds.), Event-related brain potentials: Basic issues and applications (pp. 19-36). New York: Oxford University Press.

OSTERHOUt, L., \& HolcomB, P. J. (1995). Event-related potentials and language comprehension. In M. Rugg \& M. G. H. Coles (Eds.), Electrophysiology of mind (pp. 171-215). Oxford: Oxford University Press.

PaChella, R. G. (1974). The interpretation of reaction time in information processing research. In B. Kantowitz (Ed.), Human information processing: Tutorials in performance and cognition (pp. 41-82). New York: Halstead Press.

PaIvio, A. (1990). Mental representations: A dual coding approach. New York: Oxford University Press.

PAIvIO, A. (1991). Dual coding theory: Retrospect and current status. Canadian Journal of Psychology, 45, 255-287. 
Posner, M. I., \& Raichle, M. E. (1994). Images of mind. New York: Scientific American Library.

RATCLIFF, R. (1988). Continuous versus discrete information processing: Modeling accumulation of partial information. Psychological Review, 95, 238-255.

RATCLIFF, R., \& MCKOON, G. (1982). Speed and accuracy in the processing of false statements about semantic information. Journal of Experimental Psychology: Learning, Memory, \& Cognition, 8, 16-36.

REGAN, D. (1989). Human electrophysiology: Evoked potentials and evoked magnetic fields in science and medicine. New York: Elsevier.

Rips, L. J., SMITH, E. E., \& Shoben, E. J. (1975). Set-theoretic and network models reconsidered: A comment on Hollan's "Features and semantic memory." Psychological Review, 82, 156-157.

Rosch, E. H. (1975). Cognitive representations of semantic categories. Journal of Experimental Psychology, 104, 192-233.

RuGG, M. D. (1990). Event-related brain potentials dissociate repetition effects of high- and low-frequency words. Memory \& Cognition, $18,367-379$.

RugG, M. D., Furda, J., \& LoRIST, M. (1988). The effects of task on the modulation of event-related potentials by word repetition. Psychophysiology, 25, 55-63.

Rumelhart, D. E., McClelland, J. L., \& the PDP Research Group (1986). Parallel distributed processing: Explorations in the microstructure of cognition (Vol. 1). Cambridge, MA: MIT Press.

Saffran, E. M., \& Schwartz, M. F. (1994). Of cabbages and things: Semantic memory from a neuropsychological perspective-A tutorial review. In C. Umiltà \& M. Moscovitch (Eds.), Attention and performance $X V$ : Conscious and nonconscious information processing (pp. 508-536). Cambridge, MA: MIT Press.

SCHWANENFLUGEL, P. J. (1991). Why are abstract concepts hard to understand? In P. J. Schwanenflugel (Ed.), The psychology of word meanings (pp. 223-250). Hillsdale, NJ: Erlbaum.

Schwanenflugel, P. J., \& Shoben, E. J. (1983). Differential context effects in the comprehension of abstract and concrete verbal materials. Journal of Experimental Psychology: Learning, Memory, \& Cognition, 9, 82-102.

SCHWANENFlugel, P. J., \& STOWE, R. W. (1989). Context availability and the processing of abstract and concrete words in sentences. Reading Research Quarterly, 24, 114-126.

SCHWARTZ, T., HolcomB, P., \& Kounios, J. (1990). Semantic satiation and event-related potentials [Abstract]. The Clinical Neuropsychologist, 4, 303.

SCHWEICKERT, R. (1993). Information, time, and the structure of mental events: A 25 year review. In D. E. Meyer \& S. Kornblum (Eds.), Attention and performance XIV: Synergies in experimental psychology, artificial intelligence, and cognitive neuroscience $-A$ silver $j u$ bilee (pp. 535-566). Cambridge, MA: MIT Press.

SCHWEICKERT, R., \& Boggs, G. J. (1984). Models of central capacity and concurrency. Journal of Mathematical Psychology, 28, 223-281.

ShePARD, R. N., \& COOPER, L. A. (1982). Mental images and their transformations. Cambridge, MA: MIT Press.

SMITH, E. E. (1978). Theories of semantic memory. In W. K. Estes (Ed.), Handbook of learning and cognitive processes: Vol. 6. Linguistic functions in cognitive theories (pp. 1-56). Hillsdale, $\mathrm{NJ}$ : Erlbaum.

Smith, E. E., Shoben, E. J., \& RIPS, L. J. (1974). Structure and process in semantic memory: A featural model for semantic decisions. Psychological Review, 81, 214-241.

Smith, R. W., \& Kounios, J. (in press). Sudden insight: All-or-none processing revealed by speed-accuracy decomposition. Journal of Experimental Psychology: Learning, Memory, \& Cognition.

SMith, R. W., Kounios, J., \& OSTERHOUt, L. (1996). The applicability and robustness of speed-accuracy decomposition, a technique for measuring partial information. Manuscript submitted for publication.

STERNBERG, S. (1969). The discovery of processing stages: Extensions of Donders' method. In W. G. Koster (Ed.), Attention and performance II (pp. 276-315). Amsterdam: North-Holland.

Thomsen, C. J., Lavine, H., \& Kounios, J. (in press). Social value and attitude concepts in semantic memory: Relational structure, concept strength, and the fan effect. Social Cognition.
WICKELGREN, W. (1977). Speed-accuracy tradeoff and information processing dynamics. Acta Psychologica, 41, 67-85.

WoNG, P. K. H. (1991). Introduction to brain topography. New York: Plenum.

\section{NOTES}

1. For two reasons, this discussion will not address results from lexical decision and word-naming studies. First, since these tasks can, in principle, be performed without accessing stored semantic information, it is unclear how to make inferences about semantic memory structure on the basis of such results. Second, there is no clear evidence that the lexical store tapped by such tasks is the same as (or a subset of) semantic memory. In fact, some evidence suggests that there may be separate semantic and lexical stores (see Collins \& Loftus, 1975, for a discussion). For these reasons, this discussion will focus primarily on examples drawn from the semantic verification literature that constitutes the traditional domain of semantic memory research.

2. Some potential criticisms may apply to Lorch's (1982) study. For example, neither word frequency nor familiarity (McCloskey, 1980) were controlled for in constructing the stimulus set. Lorch provided arguments against the notion that word frequency had an important impact on the findings, though he did not discuss the familiarity issue. In addition, Casey and Heath (1990) have pointed out that their main criticism of Corbett and Wickelgren's (1978) speed-accuracy tradeoff study (see section below on speed-accuracy tradeoff) may also be applicable to Lorch's study - namely, that differences in the asymptotic level of the priming functions may be due to the inclusion of category-exemplar stimulus pairs of uncertain truth value in the low instance-dominance condition, and not that low instance-dominance pairs of certain truth value achieve lower asymptotic levels of activation.

3. Although additive-factors logic has been criticized for its dependence on the strong assumption of independent, serial processing stages (see, e.g., McClelland, 1979), it should be emphasized that the present arguments are not dependent on additive-factors logic. As argued below, the point is that such results are highly suggestive because they are consistent with a considerable body of evidence obtained from a broad variety of experimental techniques.

4. The exact relationship between typicality (E. E. Smith et al., 1974) and instance dominance (Battig \& Montague, 1969; Casey, 1992; Chumbley, 1986) is uncertain, although it has been shown that these two variables are correlated (Gruenenfelder, 1986; Larochelle \& Pineau, 1994; Mervis, Catlin, \& Rosch, 1976).

5 . There are, however, some limits to the generalizability of the findings of Kounios et al. (1987) to Casey and Heath's (1990) task. Kounios et al. presented subjects with three types of universally quantified sentences for verification: "subset," "superset," and "disjoint" (see Meyer, 1970). Casey and Heath presented their subjects with exemplar-category pairs, with a $250-\mathrm{msec}$ SOA between the two terms. The differences between the stimuli and methods of presentation utilized by Kounios et al. and Casey and Heath may have had unknown effects on the verification processes subjects used. By way of contrast to Casey and Heath, Corbett and Wickelgren (1978) used category-exemplar pairs in their task. This order of presentation is logical, since they were looking at the effects of instance dominance on verification performance, and not category dominance. It is not clear from Casey and Heath's report why they reversed this order of presentation.

6. As mentioned, the study by Thomsen et al. (in press) also yielded evidence consistent with the notion of two independent stages. In this study, however, both stages were apparently manipulable. Nevertheless, this finding should not be construed as evidence against the general point that the duration of the hypothesized early stage is invariant in the other studies described, since the other studies all required subjects to verify class-inclusion statements, while the Thomsen et al. study required subjects to make relatedness judgments.

7. The term topographic distribution refers to the fact that measured ERP component amplitudes are sometimes larger at some scalp locations than at others. If enough electrode sites are used, topographic maps can be constructed showing the distribution of these amplitudes 
across electrode sites. Such distributional evidence can provide a basis for inferences about the spatial location and orientation of the neural generators producing these components (Wong, 1991).

8. There are two main possibilities: (1) The additive amplitudes in the 150 - to $300-\mathrm{msec}$ window could be produced by two or more neural generators composed of nonoverlapping populations of neurons whose electric fields sum linearly (Nunez, 1990), or (2) the additive amplitudes were produced by a single neural generator whose output was a linear function of the outputs of two independent and nonoverlapping populations of neurons, one processing context and the other processing characteristics of the final word in isolation. In the second case, the two neuronal populations providing inputs to the mechanism that computes a linear function of these amplitudes could theoretically be operating either before or during the 150 - to $300-\mathrm{msec}$ time window. In addition, these hypothetical independent mechanisms could be insulated from the summing process by one or more mediating processes.

There is, however, a third logical possibility, namely, that the additive ERP amplitudes are produced by a single neural generator that takes inputs from two or more overlapping, nonindependent, neuronal populations and performs some type of computation that yields additive ERP amplitudes as a byproduct. Since it is not clear what sort of mechanism could accomplish this, this scenario must, at present, be considered rather improbable.

It should also be noted that just as ERP amplitude additivity suggests modularity, so amplitude interactions indicate that overlapping sets of neurons are involved. For instance, if neurons in Processor $A$ are influenced by neurons in Processor B (while performing a specific task), then the influencing neurons are, by definition, a part of Processor $A$ as well as B (i.e., while performing that task). Hence there is overlap in the sets of neurons. Conversely, amplitude additivity resulting from separate neural generators indicates that there is no mutual influence between these processors.

9. This explanation for the lack of effects for congruent sentences might, at first, seem to contradict the explanation just given for the anomalous sentence results: If ERPs reflect only brain-state changes that are time-locked to a discrete event, then how could there be a P2 effect of sentence stem type that is independent of final-word type? The answer to this question is that the P2 effect of stem type is only independent of the type of incongruent final word presented, and is not independent of the presentation of the incongruent final word itself. For example, the presentation of a final word could invoke or activate the prior stem context (resulting in an ERP deflection) in the same way irrespective of whether it is a concrete or abstract word.

10. Although in the present discussion it is assumed that there is some mapping relating "units" to neurons, no particular assumptions are made here concerning the nature of this mapping. So, a unit may correspond to a single neuron or to a population of neurons.

11. It is possible, however, that certain types of internal organization can exist for only specific types of primitive units. If this can be shown to be more than a theoretical possibility, then macrostructure does have implications for nature of the representational medium.

(Manuscript received August 30, 1993; revision accepted for publication March 26, 1996.) 